Pacific Journal of Mathematics

REFINEMENTS FOR INFINITE DIRECT DECOMPOSITIONS 


\title{
REFINEMENTS FOR INFINITE DIRECT DECOMPOSITIONS OF ALGEBRAIC SYSTEMS
}

\author{
Peter Crawley and Bjarni Jónsson
}

Introduction. An operator group with a principal series can obviously be written as a direct product of finitely many directly indecomposable admissible subgroups, and the classical WedderburnRemak-Krull-Schmidt Theorem asserts that this representation is unique up to isomorphism. Numerous generalizations of this theorem are known in the literature. ${ }^{1}$ Thus it follows from results in Baer $[1,2]$ that if the admissible center of an operator group $G$ satisfies the minimal and the local maximal conditions, then any two direct decompositions of $G$ (with arbitrarily many factors) have isomorphic refinements. In a different direction, it is shown in Crawley [4] that if an operator group $G$ has a direct decomposition each factor of which has a principal series, then any two direct decompositions of $G$ have isomorphic refinements.

The results of this paper yield sufficient conditions for a group (with or without operators) to have the isomorphic refinement property. For operator groups a common generalization of the theorems mentioned above is obtained: If an operator group $G$ has a direct decomposition such that the admissible center of each factor satisfies the minimal and local maximal conditions, then any two direct decompositions of $G$ have centrally isomorphic refinements. For groups without operators we obtain the following result which eliminates any assumption of chain conditions: If a group $G$ (without operators) has a direct decomposition such that the center of each factor is countable and the reduced part of the center of each factor is a torsion group with primary components of bounded order, then any two direct decompositions of $G$ have centrally isomorphic refinements.

Actually our results hold for a much wider class of algebraic structures, namely for algebras in the sense of Jónsson-Tarski [6], and it is in this more general framework that the theory is developed. The terminology from general algebra used in this preliminary discussion will be explained in $\S 1$.

Our techniques are based on an exchange property defined as follows: An algebra $B$ is said to have the exchange property if, for

Received August 27, 1963. This work was supported in part by NSF Grants G-17957 and G-19673. A summary of the results presented here has appeared in Bull. Amer. Math. Soc 69 (1963), 541-547.

1 For a fairly complete list of references see Baer [1, 2] or Specht [8], p. 449. 
any algebras $A, C$ and $D_{i}(i \in I)$, the condition

$$
A=B \times C=\prod_{i \in I} D_{i}
$$

implies that there exist subalgebras $E_{i} \subseteq D_{i}(i \in I)$ such that

$$
A=B \times \prod_{i \in I} E_{i} .
$$

The principal result relating this notion to the isomorphic refinement problem is Theorem 7.1, which asserts that if an algebra $A$ is a direct product of subalgebras each of which has the exchange property and has a countable generated center, then any two direct decompositions of $A$ have centrally isomorphic refinements. Two related results are obtained where no cardinality conditions are imposed on the centers, but the decompositions involved are of a more special nature. First (4.2), if $A=B_{0} \times B_{1} \times B_{2} \times \cdots=C_{0} \times C_{1} \times C_{2} \cdots$, with countably many factors, and if all the subalgebras $B_{i}$ and $C_{j}$ have the exchange property, then these two direct decompositions have centrally isomorphic refinements. Second (5.3), if $A$ is a direct product of subalgebras each having the exchange property, then any two direct decompositions of $A$ into indecomposable factors are centrally isomorphic.

In $\S \S 8-11$ sufficient conditions are given in order for an algebra $B$ to have the exchange property. In $\S 8$ it is shown that if the center $B^{c}$ of $B$ has the exchange property, then so does $B$. There it is also shown that in proving the exchange property for an algebra $B$ we may assume that the factors $D_{i}$ are isomorphic to subalgebras of $B$. In $\S 9$ we prove that if $B^{c}$ satisfies the minimal and local maximal conditions, then $B$ has the exchange property and $B^{c}$ is countably generated. Sections 10 and 11 are devoted to the study of binary algebras (algebras with just one operation, the binary operation + ). The main result here (11.5) asserts that if the reduced part of the abelian group $B^{c}$ is a torsion group all of whose primary components are torsion-complete, then $B$ has the exchange property. In the twelfth and final section some counterexamples and open problems are discussed.

1. Fundamental concepts. Our terminology is largely the same as that in Jónsson-Tarski [6], and it will therefore be described very briefly. By an algebra we shall mean a system consisting of a set $A$, a binary operation + called addition, a distinguished element 0 called the zero element of the algebra, and operations $F_{t}(t \in T)$ each of which is of some finite $e^{2}$ rank $\rho(t)$, subject only to the following conditions:

${ }^{2}$ In Jónsson-Tarski [6] the operations are not required to be of finite rank. The main reason for this restriction is that it insures that the center of an algebra is a central subalgebra. 
(i) $A$ is closed under the operation + and the operations $F_{t}(t \in T)$;

(ii) for all $x \in A, x+0=0+x=x$;

(iii) $F_{t}(0,0, \cdots, 0)=0$ for all $t \in T$.

The set $T$ and the function $\rho$ are assumed to be the same for all the algebras under consideration. We shall identify the algebras with the sets of all their elements, and shall in general use the same symbols, ,$+ F_{t}$ and 0 , to denote the operations and the zero elements of all the algebras. If no auxiliary operations $F_{t}$ are present, i.e. if $T=\varnothing$, then we refer to $A$ as a binary algebra.

An obvious example of an algebra is an operator group, i.e. an algebra for which addition is associative, each element has an additive inverse, and each $F_{t}(t \in T)$ is a unary operation which distributes with respect to + . Similarly, an ordinary group without operators is a binary algebra.

If $A$ is an algebra, then the sum of finitely many elements $x_{0}, x_{1}, \cdots, x_{k}, \cdots \in A$ is defined recursively by

$$
\sum_{k<0} x_{k}=0 ; \quad \sum_{k<n+1} x_{k}=\sum_{k<n} x_{k}+x_{n} \quad(n=0,1, \cdots) .
$$

It is convenient to define also the (un-ordered) sum of certain special systems of elements $x_{i} \in A(i \in I)$. This sum is defined if and only if there exist finitely many distinct elements $i_{0}, i_{1}, \cdots, i_{n-1} \in I$ such that $x_{i}=0$ whenever $i \in I-\left\{i_{0}, i_{1}, \cdots, i_{n-1}\right\}$ and such that

$$
\sum_{k<n} x_{k}=\sum_{k<n} x_{i_{\varphi(k)}}
$$

for every permutation $\varphi$ of the integers $0,1, \cdots, n-1$. Under these conditions we let

$$
\sum_{i \in I} x_{i}=\sum_{i<n} x_{i_{k}}
$$

For brevity, a system of elements $x_{i} \in A(i \in I)$ will be said to be finitely nonzero if there are only finitely. many indices $i \in I$ such that $x_{i} \neq 0$.

The notions of subalgebra, homomorphism, isomorphism, and congruence relation are assumed to be known. If $\theta$ is a congruence relation over an algebra $A$, then for $x \in A$ we let $x / \theta$ be the congruence class to which $x$ belongs, and for $X \subseteq A$ we let $X / \theta=\{x / \theta \mid x \in X\}$. In particular, $A / \theta$ is the quotient algebra of $A$ modulo $\theta$. Observe also that if $B$ is a subalgebra of $A$, then $B / \theta$ is a subalgebra of $A / \theta$. It should be noted that if $\theta^{\prime}$ is the restriction of $\theta$ to $B$, then $B / \theta$ and $B / \theta^{\prime}$ are in general distinct algebras although they are isomorphic.

A subalgebra $B$ of an algebra $A$ is called a subtractive subalgebra of $A$ if it satisfies the following condition: If $a \in A$ and $b \in B$, and if either $a+b \in B$ or $b+a \in B$, then $a \in B$.

By a central subalgebra of $A$ we mean a subalgebra $C$ of $A$ 
satisfying the following conditions:

(i) for each $c \in C$ there exists $\bar{c} \in C$ such that $c+\bar{c}=0$;

(ii) if $c \in C$ and $x, y \in A$, then $x+(y+c)=(x+c)+y=(x+y)+c$;

(iii) if $c \in C, t \in T, k<\rho(t)$, and $x_{0}, x_{1}, \cdots, x_{\rho(t)-1} \in A$, then

$$
\begin{aligned}
& F_{t}\left(x_{0}, x_{1}, \cdots, x_{k-1}, x_{k}+c, x_{k+1}, \cdots, x_{\rho(t)-1}\right) \\
&= F_{t}\left(x_{0}, x_{1}, \cdots, x_{k-1}, x_{k}, x_{k+1}, \cdots, x_{\rho(t)-1}\right) \\
&+F_{t}(0,0, \cdots, 0, c, 0, \cdots, 0) .
\end{aligned}
$$

$k$ th

It is easy to see that the family of all central subalgebras of an algebra $A$ is a complete sublattice of the lattice of all subalgebras of $A$. In particular, the union of all the central subalgebras of $A$ is a central subalgebra of $A$. This largest central subalgebra of $A$ is called the center of $A$, and is denoted by $A^{c}$. It is clear that if $A$ is an operator group, then $A^{c}$ is the usual group-theoretic admissible center of $A .^{3}$ For a binary algebra $A$ we can alternatively define the center of $A$ as the set of all those elements of $A$ that have an additive inverse and that commute and associate with all the elements of $A$. If an algebra $A$ is such that $A^{c}=A$, then we say that $A$ is abelian.

Given two subalgebras $B$ and $C$ of an algebra $A$, a function $f$ is called a central isomorphism of $B$ onto $C$,-in symbols $f: B \cong{ }^{c} C$,-if $f$ is an isomorphism of $B$ onto $C$ and for each $x \in B$ there exists $c \in A^{c}$ such that $f(x)=x+c$. We say that $B$ and $C$ are centrally isomorphic, -in symbols $B \cong{ }^{c} C$,-if there exists a central isomorphism of $B$ onto $C$.

By the outer direct product $t^{4}$ of a system of algebras $A_{i}(i \in I),-$ in symbols

$$
\prod_{i \in I}^{o} A_{i}
$$

-we mean the algebra consisting of all functions $x$ such that the domain of $x$ is $I, x(i) \in A_{i}$ for all $i \in I$, and $x(i)=0$ for all but finitely many $i \in I$. The operations in this algebra are defined componentwise, $(x+y)(i)=x(i)+y(i)$ and

$$
F_{t}\left(x_{0}, x_{1}, \cdots, x_{\rho(t)-1}\right)(i)=F_{t}\left(x_{0}(i), x_{1}(i), \cdots, x_{\rho(t)-1}(i)\right),
$$

and its zero element is the function that associates with each index $i$

${ }^{3}$ C.f., Specht [8], p. 118; here it is called the $\Omega$-center.

4 Sometimes the outer direct products are referred to as weak outer direct products, and the Cartesian products (which are used only incidentally in this paper) are called strong outer direct products. In other cases, especially in the theory of abelian groups, outer direct products are called direct sums and Cartesian products are called direct products. 
the zero element of the corresponding algebra $A_{i}$.

The concept of an algebra is designed to make it possible to introduce the notion of an inner direct product of subalgebras of an algebra $A$, and to reduce the study of (isomorphic) representations of subalgebras $B$ of $A$ as outer direct products to considerations involving this new concept. Since the notions of outer and inner direct products are often confused in the literature, and in other cases the connection between the two concepts is not clearly stated, it is perhaps worthwhile to formulate this relationship in some detail. The basic idea is, of course, that given a representation

$$
f: B \cong \prod_{i \in I}^{\circ} C_{i},
$$

we can associate with each index $i \in I$ a subalgebra $B_{i}$ of $B$ that is isomorphic to $C_{i}$. By definition, this subalgebra consists of all those elements $x \in B$ such that $f(x)(j)=0$ for all $j \in I-\{i\}$. If a system of subalgebras $B_{i}(i \in I)$ of $B$ corresponds in this manner to a representation of $B$ as an outer direct product, then we say that $B$ is an inner direct product of the subalgebras $B_{i}(i \in I)$. To complete the transition from outer direct products to inner direct products we must find out to what extent the subalgebras determine the representation, and we must formulate intrinsic necessary and sufficient conditions for $B$ to be an inner direct product of a given system of subalgebras.

The solution of the first problem is easy: two representations,

$$
f: B \cong C=\prod_{i \in I}^{o} C_{i} \text { and } f^{\prime}: B \cong C^{\prime}=\prod_{i \in I}^{o} C_{i}^{\prime}
$$

yield the same system of subalgebras $B_{i}(i \in I)$ if and only if there exist isomorphisms $g_{i}: C_{i} \cong C_{i}^{\prime}$, for all $i \in I$, such that $f^{\prime}=g f$ where the isomorphism $g: C \cong C^{\prime}$ is induced by the isomorphisms $g_{i}(i \in I)$ in the sense that $g(x)(i)=g_{i}(x(i))$ for all $x \in C$ and $i \in I$.

Regarding the second problem, we first observe that $B$ is an inner direct product of subalgebras $B_{i}(i \in I)$ of $A$ if and only if, for every element $x$ of the algebra

$$
\bar{B}=\prod_{i \in I}^{\circ} B_{i}
$$

the sum $\sum_{i \in I} x(i)$ exists, and the mapping $x \rightarrow \sum_{i \in I} x(i)$ is an isomorphism of $\bar{B}$ onto $B$.

Consider now a system of subalgebras $B_{i}(i \in I)$ of $A$, and define $\bar{B}$ as above. In order for the indicated map to be everywhere defined and to be an isomorphism of $\bar{B}$ into $A$ it is obviously necessary and sufficient that the following four conditions be satisfied: 
( I ) For any finitely nonzero system of elements $a_{i} \in B_{i}(i \in I)$, the sum $\sum_{i \in I} a_{i}$ exists.

(II) For any two finitely nonzero systems of elements $a_{i}, b_{i} \in B_{i}(i \in I)$, if $\sum_{i \in I} a_{i}=\sum_{i \in I} b_{i}$, then $a_{i}=b_{i}$ for all $i \in I$.

(III) For any two finitely nonzero systems of elements $a_{i}, b_{i} \in B_{i}(i \in I)$,

$$
\sum_{i \in I}\left(a_{i}+b_{i}\right)=\sum_{i \in I} a_{i}+\sum_{i \in I} b_{i}
$$

(IV) For any $t \in T$, and for any finitely nonzero systems of elements $a_{k, i} \in B_{i}(i \in I), k=0,1, \cdots, \rho(t)-1$,

$$
F_{t}\left(\sum_{i \in I} a_{0, i}, \cdots, \sum_{i \in I} a_{\rho(t)-1, i}\right)=\sum_{i \in I} F_{t}\left(a_{0, i}, \cdots, a_{\rho(t)-1, i}\right) .
$$

Consequently, in order that there exists a subalgebra $B$ of $A$ such that $B$ is an inner direct product of the algebras $B_{i}(i \in I)$, it is necessary and sufficient that (I)-(IV) hold. Furthermore, if such an algebra $B$ exists, then it is unique and can be characterized by either one of the following conditions:

(V) $B$ is the set of all elements $b \in A$ such that $b=\sum_{i \in I} a_{i}$ for some finitely nonzero system of elements $a_{i} \in B_{i}(i \in I)$.

( $\left.\mathrm{V}^{\prime}\right) \quad B$ is the subalgebra of $A$ generated by the union of all the algebras $B_{i}(i \in I)$.

The conditions (I)-(V) or (I)-(IV) and $\left(\mathrm{V}^{\prime}\right)$ are often taken as the definition of the phrase "the subalgebra $B$ of $A$ is the inner direct product of the subalgebra $B_{i}(i \in I)$ of $A . "$

Since we shall henceforth be concerned exclusively with inner direct products we will refer to these simply as direct products. The direct product of a system of subalgebras $B_{i}(i \in I)$ of an algebra $A$ will be denoted by

$$
\prod_{i \in I} B_{i},
$$

and the direct product of finitely many subalgebras $B_{0}, B_{1}, \cdots, B_{n-1}$ will also be written

$$
B_{0} \times B_{1} \times \cdots \times B_{n-1} .
$$

In the finite case our notion obviously coincides with the direct product in Jónsson-Tarski [6], where this notion is defined recursively in terms of the binary operation $\times$.

A subalgebra $C$ of an algebra $B$ is called a factor of $B$ if $B=C \times D$ 
for some algebra $D . \quad B$ is said to be indecomposable if it has at least. two elements and the only factors of $B$ are $B$ and $\{0\}$. By a direct decomposition or, briefly, a decomposition of $B$ we mean a representation of $B$ as a direct product of subalgebras. The direct decompositions. of $B$,

$$
B=\prod_{i \in I} C_{i}=\prod_{j \in J} D_{j},
$$

are said to be (centrally) isomorphic if there exists a one-to-one mapping $f$ of $I$ onto $J$ such that, for each $i \in I, C_{i}$ and $D_{f(i)}$ are (centrally) isomorphic. Finally, the second decomposition is said to be a refinement of the first if for each $j \in J$ there exists $i \in I$ such that $D_{j} \subseteq C_{i}$.

2. Elementary properties of direct products. In this section several simple properties of direct products are listed. Since many of these results are already known from the literature (c.f. Jónsson-Tarski [6]), and the derivations of the remaining ones offer no difficulty, all proofs will be omitted.

We assume throughout this section that $A$ is an algebra.

Lemma 2.1. If $B$ and $C$ are subalgebras of $A$ such that $B \times C$ exists, then for all $b, b^{\prime} \in B$ and $c \in C$,

$$
b+c=c+b \text { and }\left(b+b^{\prime}\right)+c=b+\left(b^{\prime}+c\right)=(b+c)+b^{\prime} .
$$

Lemma 2.2. Every factor of $A$ is a subtractive subalgebra of $A$.

Lemma 2.3. (The modular law) Suppose $B$ and $C$ are subalgebras of $A$ such that $B \times C$ exists, and suppose $D$ is a subtractive subalgebra of $A$. If $B \subseteq D$, then $(B \times C) \cap D=B \times(C \cap D)$. In. particular, if $B \subseteq D \leqq B \times C$, then $D=B \times(C \cap D)$.

Lemma 2.4. If, for each $i \in I, B_{i}$ and $B_{i}^{\prime}$ are subalgebras of $A$. such that $B_{i}^{\prime} \subseteq B_{i}$, and if the direct product

$$
B=\prod_{i \in I} B_{i}
$$

exists, then

(i) the direct product

$$
B^{\prime}=\prod_{i \in I} B_{i}^{\prime}
$$

exists and is a subalgebra of $B$. 
(ii) $B^{\prime}=B$ if and only if $B_{i}^{\prime}=B_{i}$ for all $i \in I$.

(iii) $B^{\prime}$ is a subtractive subalgebra of $B$ if and only if, for each $i \in I, B_{i}^{\prime}$ is a subtractive subalgebra of $B_{i}$.

(iv) $B^{\prime}$ is a central subalgebra of $A$ if and only if, for each $i \in I$, $B_{i}^{\prime}$ is a central subalgebra of $B_{i}$.

Lemma 2.5. Suppose $B_{i}(i \in I)$ are subalgebras of $A$. Then

$$
A=\prod_{i \in I} B_{i}
$$

if and only if there exist homomorphisms $f_{i}$ of $A$ onto $B_{i}$, for all $i \in I$, such that for each $a \in A$

$$
a=\sum_{i \in I} f_{i}(a) \text {, and } f_{i} f_{j}(a)=0 \text { whenever } i, j \in I \text { and } i \neq j .
$$

These homomorphisms $f_{i}$, if they exist, are unique and have the property that $f_{i} f_{i}=f_{i}$ for all $i \in I$.

Definition 2.6. Assuming that

$$
A=\prod_{i \in I} B_{i},
$$

the homomorphisms $f_{i}$ characterized by the conditions in Lemma 2.5 are called the projections of $A$ onto the algebras $B_{i}$ induced by the given decomposition of $A$.

Lemma 2.7. Suppose $B_{i}(i \in I)$ are subalgebras of $A$. Then the direct product

$$
\prod_{i \in I} B_{i}
$$

exists if and only if for each finite subset $J$ of $I$ the direct product

$$
\prod_{i \in J} B_{i}
$$

exists.

LemMA 2.8. Suppose that $B_{i}(i \in I)$ are subalgebras of $A$, that $I=\bigcup_{k \in K} J_{k}$, and that the sets $J_{k}(k \in K)$ are pairwise disjoint. If either the direct product

$$
B=\prod_{i \in I} B_{i}
$$

exists, or if the direct products

$$
C_{k}=\prod_{i \in J_{k}} B_{i}(k \in K) \quad \text { and } \quad B^{\prime}=\prod_{k \in K} C_{k}
$$

exists, then all these direct products exist, and $B=B^{\prime}$. 
Lemma 2.9. Given two direct decompositions of $A$,

$$
A=\prod_{i \in I} B_{i} \text { and } A=\prod_{j \in J} C_{j},
$$

the second decomposition of $A$ is a refinement of the first if and only if for each $i \in I$ there exists a subset $J_{i}$ of $J$ such that

$$
B_{i}=\prod_{j \in J_{i}} C_{j}
$$

LEMMA 2.10. If $B_{i}(i \in I)$ are subalgebras of $A$, if the direct product

$$
\prod_{i \in I} B_{i}
$$

exists, and if $J$ and $K$ are subsets of $I$, then

$$
\left(\prod_{i \in J} B_{i}\right) \cap\left(\prod_{i \in K} B_{i}\right)=\prod_{i \in J \cap K} B_{i}
$$

Lemma 2.11. Suppose $B_{i}(i \in I)$ are subalgebras of $A$, and for each $i \in I$ let $\bar{B}_{i}$ be the subalgebra of $A$ that is generated by the union of all the algebras $B_{j}$ with $j \in I$ and $i \neq j$. Then the direct product

$$
\prod_{i \in I} B_{i}
$$

exists if and only if $B_{i} \times \bar{B}_{i}$ exists for all $i \in I$.

Lemma 2.12. If $C$ is a central subalgebra of $A$, then for all $a, a^{\prime} \in A$ and $c \in C$,

$$
a+c=c+a \text {, and } a+c=a^{\prime}+c \text { implies } a=a^{\prime} .
$$

Lemma 2.13. If $C$ is a central subalgebra of $A$, then $C$ is a subtractive subalgebra of $A$, and $C$ is an abelian group under the operation + .

LEMMA 2.14. If $B$ is a subtractive subalgebra of $A$, and if $C$ is a central subalgebra of $A$, then

(i) $B \cap C$ is a central subalgebra of $A$.

(ii) $B \times C$ exists if and only if $B \cap C=\{0\}$.

LEMma 2.15. Suppose $C_{0}, C_{1}, \cdots, C_{n-1}$ are central subalgebras of $A$, and for $k=1,2, \cdots, n-1$ let $\bar{C}_{k}$ be the subalgebra of $A$ that is generated by the union of the algebras $C_{0}, C_{1}, \cdots, C_{k-1}$. Then the 
direct product

$$
\prod_{k<n} C_{k}
$$

exists if and only if $C_{k} \cap \bar{C}_{k}=\{0\}$ for $k=1,2, \cdots, n-1$.

LEMMA 2.16. If

$$
A=\prod_{i \in I} B_{i}
$$

then

$$
A^{c}=\prod_{i \in I} B_{i}^{c}
$$

LEMMA 2.17. Suppose

$$
A=\prod_{i \in I} B_{i}=\prod_{j \in J} C_{j},
$$

and for $i \in I$ and $j \in J$ let $f_{i}$ and $g_{j}$ be the projections of $A$ onto $B_{i}$ and onto $C_{j}$ that are induced by these two decompositions. If $i, i^{\prime} \in I$, $j \in J$, and $i \neq i^{\prime}$, then $f_{i} g_{j} f_{i}$, maps $A$ into the center of $B_{i}$.

Lemma 2.18. If

$$
A=B \times C=\prod_{i \in I} D_{i},
$$

then

$$
B^{c} \times C=\prod_{i \in I}\left(\left(B^{c} \times C\right) \cap D_{i}\right)
$$

Lemma 2.19. If $B, C$ and $D$ are subalgebras of $A$ such that $B \times C$ exists, then the conditions

$$
B \times C=B \times D \text { and } B^{c} \times C=B^{c} \times D
$$

are equivalent.

Lemma 2.20. Suppose $A=B \times C=B \times D$, and let $f$ and $g$ be the projections of $A$ onto $C$ and onto $D$ induced by these two decompositions. Then the restriction $g^{\prime}$ of $g$ to $C$ is a central isomorphism of $C$ onto $D$, and the inverse of $g^{\prime}$ is equal to the restriction of $f$ to $D$.

3. Exchange properties. The central concept of this paper, the exchange property, was mentioned in the introduction. We now formulate this notion more precisely. 
DeFinition 3.1. Given a cardinal $m$, an algebra $B$ is said to have the m-exchange property if for any algebra $A$ containing $B$ as a subalgebra, and for any subalgebras $C$ and $D_{i}(i \in I)$ of $A$, where the cardinal of $I$ does not exceed $m$, the condition

$$
A=B \times C=\prod_{i \in I} D_{i}
$$

implies that there exist subalgebras $E_{i} \subseteq D_{i}(i \in I)$ such that

$$
A=B \times \prod_{i \in I} E_{i} .
$$

We say that $B$ has the exchange property if it has the m-exchange property for every cardinal $m$. We say that $B$ has the finite exchange property if it has the m-exchange property for every finite cardinal $m$.

It would be of some interest to know whether, for two given cardinals $m$ and $n$ with $1<m<n$, the $m$-exchange property implies the $n$-exchange property. It will be shown later in this section that this is the case whenever $n$ is finite, whence it follows that the 2-exchange property implies the finite exchange property. In all other cases the answer is unknown. However, since every algebra that is known to have the 2-exchange property is also known to have the exchange property, this question is not crucial at the present.

This section will be devoted to a series of lemmas involving or relating to the exchange properties that will be used in the subsequent sections

Definition 3.2. A congruence relation $\theta$ over an algebra $A$ is said to be consistent with a decomposition

$$
A=\prod_{i \in I} B_{i}
$$

of $A$ if, for all $x, y \in A$ and $i \in I$,

$$
x \theta y \text { implies } f_{i}(x) \theta f_{i}(y),
$$

where $f_{i}$ is the projection of $A$, onto $B_{i}$ induced by the given decomposition.

If $A$ is a group, then the congruence relation $\theta$ that corresponds to a normal subgroup $N$ of $A$ is consistent with the above decomposition of $A$ if and only if

$$
N=\prod_{i \in I}\left(B_{i} \cap N\right)
$$


For an arbitrary algebra $A$, a congruence relation $\theta$ over $A$ is easily seen to be consistent with a given decomposition of $A$ if and only if $\theta$ is generated (in an obvious sense that need not be made more precise here) by its restrictions to the factors in the decomposition.

LEMma 3.3. Suppose the congruence relation $\theta$ over the algebra $A$ is consistent with the decomposition

$$
A=\prod_{i \in I} B_{i}
$$

of A. Then

$$
A / \theta=\prod_{i \in I}\left(B_{i} / \theta\right)
$$

More generally, for any system of subalgebras $B_{i}^{\prime} \subseteq B_{i}(i \in I)$,

$$
\left(\prod_{i \in I} B_{i}^{i}\right) / \theta=\prod_{i \in I}\left(B_{i} / \theta\right) \text {. }
$$

Proof. For each $i \in I$ let $f_{i}$ be the projection of $A$ onto $B_{i}$ induced by the given decomposition of $A$. The consistency of $\theta$ is equivalent to the assertion that for each $i \in I$ there exists a map $g_{i}$ of $A / \theta$ onto $B_{i} / \theta$ such that $g_{i}(x / \theta)=f_{i}(x) / \theta$ for all $x \in A$. It is obvious that $g_{i}$ is a homomorphism. For each $x \in A$,

$$
x=\sum_{i \in I} f_{i}(x),
$$

and therefore

$$
x / \theta=\sum_{i \in I}\left(f_{i}(x) / \theta\right)=\sum_{i \in I} g_{i}(x / \theta) .
$$

Finally, if $i$ and $j$ are distinct members of $I$, then for all $x \in A$, $g_{i} g_{j}(x / \theta)=f_{i} f_{j}(x) / \theta=0 / \theta$. Hence the first part of the conclusion follows by 2.5. The second part of conclusion follows from the first part together with the observation that the algebra

$$
\left(\prod_{i \in \Lambda} B_{i}^{\prime}\right) / \theta
$$

consists of all elements

$$
\left(\sum_{i \in I} x_{i}\right) / \theta=\sum_{i \in I}\left(x_{i} / \theta\right),
$$

associated with finitely non-zero systems $x_{i} \in B_{i}(i \in I)$.

LEMma 3.4. Suppose the congruence relation $\theta$ over the algebra $A$ is consistent with the decompositions 


$$
A=B \times C=\prod_{i \in I} D_{i}
$$

of $A$, and suppose the restriction of $\theta$ to $B$ is the identity relation. If, for each $i \in I, \bar{E}_{i}$ is a subalgebra of $D_{i} / \theta$, and if

$$
A / \theta=B / \theta \times \prod_{i \in I} \bar{E}_{i},
$$

then there exist subalgebras $E_{i} \subseteq D_{i}(i \in I)$ such that $\bar{E}_{i}=E_{i} / \theta$ for all. $i \in I$ and

$$
A=B \times \prod_{i \in I} E_{i}
$$

Proof. For each $i \in I$ let $f_{i}$ be the projection of $A$ onto $D_{i}$ induced by the second of the two given decompositions of $A$. Letting

$$
A^{\prime}=B^{c} \times C,
$$

we infer from 2.18 that

$$
A^{\prime}=\prod_{i \in I} D_{i}^{\prime} \quad \text { where } D_{i}^{\prime}=A^{\prime} \cap D_{i}(i \in I) .
$$

Obviously $(B / \theta)^{c}=B^{c} / \theta$, since the restriction of $\theta$ to $B$ is the identity relation. It therefore follows by (1), (2), 3.3 and 2.19 that

$$
A^{\prime} / \theta=B^{c} / \theta \times C / \theta=\prod_{i \in I}\left(D_{i}^{\prime} / \theta\right)=\left(B^{c} / \theta\right) \times \prod_{i \in I} \bar{E}_{i} .
$$

Next observe that

$$
D_{i}^{\prime} / \theta=\left(A^{\prime} / \theta\right) \cap\left(D_{i} / \theta\right) .
$$

To prove this we use the fact that

$$
A / \theta=(B / \theta) \times(C / \theta)=\prod_{i \in I}\left(D_{i} / \theta\right)
$$

and that

$$
A^{\prime} / \theta=\left(B^{c} / \theta\right) \times(C / \theta),
$$

and we infer by 2.18 that

$$
A^{\prime} / \theta=\prod_{i \in I}\left(\left(A^{\prime} / \theta\right) \cap\left(D_{i} / \theta\right)\right) .
$$

Since in (4) the left hand side is obviously included in the right hand side, the equality follows from (3) and (5) with the aid of 2.4 (ii).

It follows from (3) and (4), together with the hypothesis $\bar{E}_{i} \subseteq D_{i} / \theta$, that

$$
\bar{E}_{i} \subseteq D_{i}^{\prime} / \theta
$$


Letting

$$
E_{i}=\left\{x \mid x \in D_{i}^{\prime} \text { and } x / \theta \in \bar{E}_{i}\right\},
$$

we see that $E_{i}$ is a subalgebra of $D_{i}^{\prime}$, and we infer from (6) that

$$
\bar{E}_{i}=E_{i} / \theta \text {. }
$$

From the fact that $D_{i}^{\prime}$ is a subtractive subalgebra of $A^{\prime}$ and that $\bar{E}_{i}$ is a subtractive subalgebra of $A^{\prime} \mid \theta$ it readily follows that $E_{i}$ is a subtractive subalgebra of $A^{\prime}$. Consequently,

$$
E=\prod_{i \in I} E_{i}
$$

is also a subtractive subalgebra of $A^{\prime}$. Furthermore, if $b \in B^{c} \cap E$, then

$$
b / \theta \in\left(B^{c} / \theta\right) \cap(E / \theta)=\{0 / \theta\},
$$

and therefore $b=0$. Thus $B^{c} \cap E=\{0\}$, and we infer by 2.14 (ii) that the direct product $B^{c} \times E$ exists, and is a subalgebra of $A^{\prime}$.

To complete the proof it suffices to show that $D_{k}^{\prime} \subseteq B^{c} \times E$ for every $k \in I$. Consider an element $x \in D_{k}^{\prime}$. By (3) and (7) there exist an element $b \in B^{c}$ and a finitely nonzero system of elements $e_{i} \in E_{i}$ such that

$$
x \theta b+\sum_{i \in I} e_{i}
$$

"There exists an element $\bar{b} \in B^{c}$ such that $b+\bar{b}=0$. Hence

$$
\bar{b}+x \theta \sum_{i \in I} e_{i} \text {. }
$$

Consequently $f_{k}(\bar{b})+x \theta e_{k}$ and $f_{i}(\bar{b})=f_{i}(\bar{b}+x) \theta e_{i}$ whenever $k \neq i \in I$. Inasmuch as

$$
\bar{b} \in B^{c} \subseteq \prod_{i \in I} D_{i}^{\prime},
$$

we infer that $f_{k}(\bar{b})+x \in E_{k}$ and that $f_{i}(\bar{b}) \in E_{i}$ whenever $k \neq i \in I$. Thus

$$
f_{k}(b)=b+\sum_{k \neq i \in I} f_{i}(\bar{b}) \in B^{c} \times E,
$$

and hence

$$
\dot{x}=f_{k}(b)+\left(f_{k}(\bar{b})+x\right) \in B^{c} \times E,
$$

as was to be shown.

LEMMA 3.5. If $B$ is a factor of an algebra $A$, then there exists 
a unique congruence relation $\theta$ over $A$ with the property that if $C$ is any subalgebra of $A$ with $A=B \times C$, and if $g$ is the projection of $A$ onto $C$ induced by this decomposition, then for all $x, y \in A$ the conditions $x \theta y$ and $g(x)=g(y)$ are equivalent.

Proof. Since the projection $g$ of $A$ onto $C$ induced by the decomposition $A=B \times C$ is a homomorphism of $A$ onto $C$, the condition

$$
x \theta y \text { if and only if } g(x)=g(y)
$$

defines a congruence relation $\theta$ over $A$. To complete the proof it therefore suffices to show that for any other decomposition $A=B \times C^{\prime}$, and the induced projection $g^{\prime}$ of $A$ onto $C^{\prime}$, the conditions $g(x)=g(y)$ and $g^{\prime}(x)=g^{\prime}(y)$ are equivalent. To see that this is true we simply observe that for all $x \in A, g^{\prime}(x)=g^{\prime} g(x)$ and $g(x)=g g^{\prime}(x)$. In fact, there exists $b \in B$ such that $x=b+g(x)$; hence

$$
g^{\prime}(x)=g^{\prime}(b)+g^{\prime} g(x)=g^{\prime} g(x) .
$$

The second formula is proved similarly

Definition 3.6. If $B$ is a factor of an algebra $A$, then the congruence relation $\theta$ characterized by the conditions in Lemma 3.5 is called the congruence relation over $A$ induced by $B$.

Corollary 3.7. Suppose $B$ and $C$ are subalgebras of an algebra A such that

$$
A=B \times C,
$$

and suppose $\theta$ is the congruence relation over $A$ induced by $B$. Then $0 / \theta=B$, and the restriction of $\theta$ to $C$ is the identity relation over $C$. Furthermore, $\theta$ is consistent with any decomposition of $A$ that is a refinement of the decomposition (i).

LemmA 3.8. If $B, C, D_{i}(i \in I)$ and $E$ are subalgebras of an algebra A such that

$$
A=B \times C \times E=\prod_{i \in I} D_{i} \times E,
$$

and if $\theta$ is the congruence relation over $A$ induced by $E$, then for any subalgebras $F_{i} \subseteq D_{i}(i \in I)$ the condition

$$
A / \theta=(B / \theta) \times \prod_{i \in I}\left(F_{i} / \theta\right)
$$

implies that 


$$
A=B \times \prod_{i \in I} F_{i} \times E .
$$

Proof. Since $E / \theta$ is the one-element algebra $\{0 / \theta\}$, we have

$$
A / \theta=(B / \theta) \times \prod_{i \in I}\left(F_{i} / \theta\right) \times(E / \theta) .
$$

Inasmuch as the restriction of $\theta$ to $B$ is the identity relation over $B$, we infer by 3.4 that there exist subalgebras $F_{i}^{\prime} \subseteq D_{i}(i \in I)$ and $E^{\prime} \subseteq E$ such that

$$
A=B \times \prod_{i \in I} F_{i}^{\prime} \times E^{\prime}
$$

and such that $F_{i} / \theta=F_{i}^{\prime} / \theta$ for all $i \in I$. Since the restriction of $\theta$ to $D_{i}$ is the identity relation over $D_{i}$, this last condition implies that $F_{i}^{\prime}=F_{i}$, and by the modular law we have

$$
E=E^{\prime} \times E^{\prime \prime} \quad \text { where } E^{\prime \prime}=E \cap\left(B \times \prod_{i \in I} F_{i}\right) .
$$

If $x \in E^{\prime \prime}$, then $x=y+z$ for some $y \in B$ and $z \in \Pi_{i \in I} F_{i}$. Hence $y / \theta+z / \theta=x / \theta=0 / \theta$, and it follows by (ii) that $y / \theta=z / \theta=0 / \theta$. Recalling that the restrictions of $\theta$ to $B$ and to $\prod_{i \in I} F_{i}$ are the identity relations over these algebras we infer that $y=z=0$, hence $x=0$. Thus $E^{\prime \prime}=\{0\}, E^{\prime}=E$, and (iii) holds.

Corollary 3.9. If $B, C, D_{i}(i \in I)$ and $E$ are subalgebras of an algebra $A$ with

$$
A=B \times C \times E=\prod_{i \in I} D_{i} \times E,
$$

and if $B$ has the m-exchange property, where $m$ is the cardinal of $I$, then there exist subalgebras $F_{i} \subseteq D_{i}(i \in I)$ such that

$$
A=B \times \prod_{i \in I} F_{i} \times E .
$$

Lemma 3.10. Suppose $m$ is a cardinal and $n$ is a positive integer, and suppose $B_{0}, B_{1}, \cdots, B_{n}$ are subalgebras of an algebra $B$ with $B=B_{0} \times B_{1} \times \cdots \times B_{n}$. Then $B$ has the m-exchange property if and only if each of the algebras $B_{k}(k=0,1, \cdots, n)$ has the m-exchange property.

Proof. It suffices to consider the case $n=1$. First suppose $B_{0}$ and $B_{1}$ have the $m$-exchange property. If $A$ is an algebra that contains $B$ as a subalgebra, if $C$ and $D_{i}(i \in I)$ are subalgebras of $A$ with

$$
A=B_{0} \times B_{1} \times C=\prod_{i \in I} D_{i},
$$


and if the cardinal of $I$ does not exceed $m$, then there exist subalgebras $E_{i} \subseteq D_{i}(i \in I)$ such that

$$
A=B_{0} \times \prod_{i \in I} E_{i} .
$$

From this and the first decomposition in (1) it follows by 3.9 that there exist subalgebras $F_{i} \subseteq E_{i}(i \in I)$ such that

$$
A=B_{0} \times B_{1} \times \prod_{i \in I} F_{i} .
$$

Thus $B$ has the $m$-exchange property.

Now suppose $B$ has the $m$-exchange property. Consider an algebra $A$ containing $B_{0}$ as a subalgebra, and subalgebras $C, D_{i}(i \in I)$ with

$$
A=B_{0} \times C=\prod_{i \in I} D_{i},
$$

and assume that the cardinal of $I$ does not exceed $m$. Replacing the given algebras, if necessary, by isomorphic copies, we may assume that there exists an algebra $A^{\prime}$ such that both $A$ and $B_{1}$ are subalgebras of $A^{\prime}$, and such that $A^{\prime}=A \times B_{1}$. Then

$$
A^{\prime}=B \times C=B_{1} \times \prod_{i \in I} D_{i} .
$$

If $m$ is infinite. then we can apply the $m$-exchange property to these two decompositions, but in order to accommodate also the finite cases we choose an element $k \in I$, and let $I^{\prime}=I-\{k\}$ and $E=B_{1} \times D_{k}$. Then

$$
A^{\prime}=B \times C=E \times \prod_{i \in I^{\prime}} D_{i} .
$$

Hence there exist subalgebras $E^{\prime} \leqq E$ and $D_{i}^{\prime} \subseteq D_{i}\left(i \in I^{\prime}\right)$ such that

$$
A^{\prime}=B \times E^{\prime} \times \prod_{i \in I^{\prime}} D_{i}^{\prime} .
$$

Since $B \times E^{\prime}$ is a factor of $A^{\prime}$, and hence a subtractive subalgebra of $A^{\prime}$, and since $B \subseteq B \times E^{\prime} \subseteq B \times D_{k}$, it follows from the modular law that $B \times E^{\prime}=B \times D_{k}^{\prime}$ where $D_{k}^{\prime}=\left(B \times E^{\prime}\right) \cap D_{k}$. Substituting this into (2) we obtain

$$
A^{\prime}=B \times \prod_{i \in I} D_{i}^{\prime}
$$

Inasmuch as

$$
A^{\prime}=B_{1} \times A=B_{1} \times\left(B_{0} \times \prod_{i \in I} D_{i}^{\prime}\right)
$$

and 


$$
B_{0} \times \prod_{i \in I} D_{k}^{\prime} \subseteq A
$$

we conclude by 2.4 that

$$
A=B_{0} \times \prod_{i \in I} D_{k}^{\prime}
$$

Thus $B_{0}$ has the $m$-exchange property.

LEMMA 3.11. If an algebra $B$ has the 2-exchange property, then $B$ has the finite exchange property.

Proof. It suffices to show, for an arbitrary integer $m>1$, that if $B$ has the $m$-exchange property, then $B$ has the $(m+1)$-exchange property. Assuming that

$$
A=B \times C=D_{0} \times D_{1} \times \cdots \times D_{m},
$$

let $E=D_{0} \times D_{1} \times \cdots \times D_{m-1}$. Then $A=B \times C=E \times D_{m}$, and since $B$ has the 2-exchange property, there exist algebras $E^{\prime} \subseteq E$ and $D_{m}^{\prime} \subseteq D_{m}$ such that $A=B \times E^{\prime} \times D_{m}^{\prime}$. Letting

$$
E^{\prime \prime}=E \cap\left(B \times D_{m}^{\prime}\right) \quad \text { and } \quad D_{m}^{\prime \prime}=D_{m} \cap\left(B \times E^{\prime}\right),
$$

we infer by the modular law that $E=E^{\prime} \times E^{\prime \prime}$ and $D_{m}=D_{m}^{\prime} \times D_{m}^{\prime \prime}$. From the decompositions

$$
A=B \times\left(E^{\prime} \times D_{m}^{\prime}\right)=\left(E^{\prime \prime} \times D_{m}^{\prime \prime}\right) \times\left(E^{\prime} \times D_{m}^{\prime}\right)
$$

we see by 2.19 that $E^{\prime \prime}$ is isomorphic to a factor of $B$. Consequently $E^{\prime \prime}$ has the $m$-exchange property by 3.10 . Since

$$
E=E^{\prime} \times E^{\prime \prime}=D_{0} \times D_{1} \times \cdots \times D_{m-1},
$$

it therefore follows that there exist subalgebras $D_{i}^{\prime} \subseteq D_{i}, i=0,1, \cdots$, $m-1$, such that

$$
E=E^{\prime \prime} \times D_{0}^{\prime} \times D_{1}^{\prime} \times \cdots \times D_{m-1}^{\prime} .
$$

Inasmuch as $E^{\prime \prime} \subseteq B \times D_{m}^{\prime} \subseteq E^{\prime \prime} \times\left(E^{\prime} \times D_{m}\right)$, and application of the modular law yields

$$
B \times D_{m}^{\prime}=E^{\prime \prime} \times E^{\prime \prime \prime} \text { where } E^{\prime \prime \prime}=\left(B \times D_{m}^{\prime}\right) \cap\left(E^{\prime} \times D_{m}\right),
$$

and we conclude that

$$
\begin{aligned}
A=E^{\prime} \times E^{\prime \prime} \times E^{\prime \prime \prime} & =E \times E^{\prime \prime \prime}=D_{0}^{\prime} \times D_{1}^{\prime} \times \cdots \times D_{m-1}^{\prime} \times E^{\prime \prime} \times E^{\prime \prime \prime} \\
& =B \times D_{0}^{\prime} \times D_{1}^{\prime} \times \cdots \times D_{m}^{\prime} .
\end{aligned}
$$

Thus $B$ has the $(m+1)$-exchange property, as was to be shown. 
LEMMA 3.12. Suppose $m$ is a cardinal greater than 1, and. suppose $B$ is an algebra whose center is generated by a set whose cardinal does not exceed $m$. If $B$ has the m-exchange property, then $B$ has the exchange property.

Proof. Assuming that

$$
A=B \times C=\prod_{i \in I} D_{i},
$$

write

$$
D_{J}=\prod_{i \in J} D_{i} \quad \text { for } J \subseteq I .
$$

Then there exists as set $J \subseteq I$ such that $B^{c} \subseteq D_{J}$, and such that $J$ is finite if $m$ is finite, and the cardinal of $J$ is at most $m$ if $m$ is infinite. By hypothesis (and by 3.11 in case $m$ is finite), there exist subalgebras. $E_{i} \subseteq D_{i}$ for all $i \in J$ and a subalgebra $F$ of $D_{I-J}$ such that

$$
A=B \times \prod_{i \in J} E_{i} \times F .
$$

Letting $E_{i}==F \cap D_{i}$ for $i \in I-J$, we shall show that

$$
F=\prod_{i \in I-J} E_{i}
$$

whence it follows that

$$
A=B \times \prod_{i \in I} E_{i} .
$$

Given $a \in F$, there exists a finitely non-zero system of elements: $d_{i} \in D_{i}(i \in I-J)$ such that

$$
a=\sum_{i \in I-J} d_{i} .
$$

Considering a fixed index $k \in I-J$, we can find elements $b \in B, e_{i} \in E_{i}$ $(i \in J)$ and $f \in F$ such that

$$
d_{k}=b+\sum_{i \in J} e_{i}+f .
$$

By 2.17, $b \in B^{c}$, hence $b \in D_{\mathrm{J}}$. Consequently the element

$$
x=b+\sum_{i \in J} e_{i}
$$

belongs to $D_{J}$. But the elements $d_{k}$ and $f$ belong to the subtractive subalgebra $D_{I-J}$ of $A$, and it follows by (2) and (3) that $x \in D_{I-J}$. Thus $x=0, d_{k}=f$, and $d_{k} \in F \cap D_{k}=E_{k}$. Since this last formula holds. for all $k \in I-J$, we conclude that

$$
a \in \prod_{i \in I-J} E_{i}
$$


From this (1) easily follows.

4. Direct decompositions with countably many factors. The next theorem and its simple proof are included primarily in order to show why a similar argument fails to apply when we drop the assumption that the set $I$ be finite.

THEOREM 4.1. If the algebra $A$ has the m-exchange property (where $m$ is some cardinal), and if

$$
A=\prod_{\imath \in I} B_{i}=\prod_{j \in J} C_{j}
$$

where the set $I$ is finite and the cardinal of $J$ does not exceed $m$, then these two direct decompositions of $A$ have centrally isomorphic refinements.

Proof. For notational convenience we assume that $I$ consists of the integers $0,1, \cdots, n$. By $3.10, B_{0}, B_{1}, \cdots, B_{n}$ have the $m$-exchange property, and by successive applications of 3.9 we obtain, for each $j \in J$, a sequence of subalgebras

$$
C_{j} \supseteqq C_{0, j}^{\prime} \supseteqq C_{1, j}^{\prime} \supseteqq \cdots \supseteqq C_{n-1, j}^{\prime} \supseteqq C_{n, j}^{\prime}=\{0\}
$$

such that

$$
A=B_{0} \times \cdots \times B_{i} \times \prod_{j \in J} C_{i, j}^{\prime} \quad(i=0,1, \cdots, n) .
$$

Since all the subalgebras $C_{i, j}^{\prime}$ are factors of $A$, it follows by the modular law that subalgebras $C_{i, j}(i=0, \cdots, n, j \in J)$ exist such that for each $j \in J$,

$$
C_{j}=C_{0, j}^{\prime} \times C_{0, j}, \quad \text { and } \quad C_{i-1, j}^{\prime}=C_{i, j}^{\prime} \times C_{i, j} \quad(i=1, \cdots, n) .
$$

Consequently

$$
C_{j}=\prod_{i \leqq n} C_{i, j} \quad(j \in J)
$$

and

$$
A=\prod_{i<p} B_{i} \times \prod_{p \leqq i \leqq n} \prod_{j \in J} C_{i, j} \quad(p=0,1, \cdots, n+1) .
$$

comparing the two decompositions obtained from this last formula by taking two successive values of $p, p=k$ and $p=k+1$, we infer by 2.20 that

$$
B_{k} \cong{ }^{c} \prod_{j \in J} C_{k, j} \quad(k=0,1, \cdots, n) ;
$$


and we conclude that $B_{k}$ has a decomposition

$$
B_{k}=\prod_{j \in J} B_{k, j} \text { with } B_{k, j} \cong{ }^{c} C_{k, j} \text { all } j \in J .
$$

Attempting to extend the above argument to the case when both $I$ and $J$ are infinite, one encounters difficulty in connection with the "passage through limits." For instance, in the simplest case, where $I$ is the set of all natural numbers, the above process yields subalgebras $C_{i, j}, C_{i, j}^{\prime}(i=0,1 \cdots, j \in J)$ with

$$
C_{j}=C_{k, i}^{\prime} \times \prod_{i \leqq k} C_{k, j} \text { and } B_{k} \cong \prod_{j \in J}^{c} C_{k, j},
$$

but it may happen that the direct product

$$
\prod_{i<\infty} C_{i, j}
$$

is a proper subalgebra of $C_{j}$. It is not known how this difficulty can be overcome in general, but we will show how it can be avoided in certain situations. For the case when $I$ and $J$ are denumerable, this is done below by a simple argument involving a diagonal process.

Observe that in the proof of 4.1 we did not make direct use of the fact that $A$ has the $m$-exchange property, but applied this property to the factors $B_{i}$. Because of the finiteness of $I$ this distinction is immaterial here, but in later results a significant generalization is obtained by assuming the exchange properties for the factors in some decomposition (or decompositions) rather than for the whole algebra. Incidentally, 4.1 could actually be generalized by observing that no use is made of the fact that $B_{n}$ has the $m$-exchange property.

THEOREM 4.2. If an algebra $A$ has two direct decompositions with countably many factors,

$$
A=B_{0} \times B_{1} \times B_{2} \times \cdots=C_{0} \times C_{1} \times C_{2} \times \cdots,
$$

where all the factors $B_{i}$ and $C_{j}$ have the $\aleph_{0}$-exchange property, then these two direct decompositions have centrally isomorphic refinements.

Proof. Since $B_{0}$ has the $\boldsymbol{\aleph}_{0}$-exchange property, there exist subalgebras $C_{0, j}, C_{0, j}^{\prime}$ with $C_{j}=C_{0, j} \times C_{0, j}^{\prime}$ for $j=0,1,2, \cdots$ such that

$$
A=B_{0} \times C_{0,0}^{\prime} \times C_{0,1}^{\prime} \times C_{0,2}^{\prime} \times \cdots,
$$

and from this it follows by 2.20 that

$$
B_{0} \cong{ }^{c} C_{0,0} \times C_{0,1} \times C_{0,2} \times \cdots .
$$

The factor $C_{0,0}^{\prime}$ of $C_{0}$ has the $\aleph_{0}$-exchange property by 3.10. Applying 
3.9 to (1) and the first decomposition in (i) we obtain subalgebras $B_{i, 0}, B_{i, 0}^{\prime}$ with $B_{i}=B_{i, 0} \times B_{i, 0}^{\prime}$ for $i=0,1,2, \cdots$ such that

$$
A=B_{0} \times C_{0,0}^{\prime} \times B_{1,0}^{\prime} \times B_{2,0}^{\prime} \times B_{3,0}^{\prime} \times \cdots,
$$

and it follows, again by 2.20 , that

$$
C_{0,0}^{\prime} \cong{ }^{c} B_{1,0} \times B_{2,0} \times B_{3,0} \times \cdots .
$$

Now, using the fact that $B_{1,0}^{\prime}$ has the $\aleph_{0}$-exchange property, we apply 3.9 to (3) and (1). This yields subalgebras $C_{1, j}, C_{1, j}^{\prime}$ with $C_{0, j}^{\prime}=$ $C_{1, j} \times C_{1, j}^{\prime}$ for $j=1,2,3, \cdots$ such that

$$
\begin{aligned}
A & =B_{0} \times C_{0,0}^{\prime} \times B_{1,0}^{\prime} \times C_{1,1}^{\prime} \times C_{1,2}^{\prime} \times C_{1,3}^{\prime} \times \cdots, \\
B_{1,0}^{\prime} & \cong{ }^{c} C_{1,1} \times C_{1,2} \times C_{1,3} \times \cdots .
\end{aligned}
$$

Next, from (4) and (3) we obtain subalgebras $B_{i, 1}, B_{i, 1}^{\prime}$ with $B_{i, 0}^{\prime}=$ $B_{i, 1} \times B_{i, 1}^{\prime}$ for $i=2,3, \cdots$ such that

$$
\begin{aligned}
A & =B_{0} \times C_{0,0}^{\prime} \times B_{1,0}^{\prime} \times C_{1,1}^{\prime} \times B_{2,1}^{\prime} \times B_{3,1}^{\prime} \times B_{4,1}^{\prime} \times \cdots, \\
C_{1,1}^{\prime} & \cong{ }^{c} B_{2,1} \times B_{3,1} \times B_{4,1} \times \cdots .
\end{aligned}
$$

Continuing in this manner we obtain subalgebras $B_{i, j}, B_{i, j}^{\prime}$ for $i>j$ and $C_{i, j}$ for $i \leqq j$ such that the following four conditions hold for $i=1,2,3, \cdots$ and $j=0,1,2, \cdots$ :

$$
\begin{aligned}
B_{i} & =B_{i, 0} \times B_{i, 1} \times \cdots \times B_{i, i-1} \times B_{i, i-1}^{\prime}, \\
C_{j} & =C_{0, j} \times C_{1, j} \times \cdots \times C_{j, j} \times C_{j, j}^{\prime}, \\
B_{i, i-1}^{\prime} & \cong{ }^{c} C_{i, i} \times C_{i, i+1} \times C_{i, i+2} \times \cdots, \\
C_{j, j}^{\prime} & \cong{ }^{c} B_{j+1, j} \times B_{j+2, j} \times B_{j+3, j} \times \cdots .
\end{aligned}
$$

From (2), (7) and (8) we infer that there exist algebras $B_{i, j}$ for $i \leqq j$ and $C_{i, j}$ for $i>j$ such that

$$
\begin{aligned}
B_{i, j} & \cong{ }^{c} C_{i, j} \text { for } i, j=0,1,2, \cdots, \\
B_{0} & =B_{0,0} \times B_{0,1} \times B_{0,2} \times \cdots, \\
B_{i, i-1}^{\prime} & =B_{i, i} \times B_{i, i+1} \times B_{i, i+2} \times \cdots \quad \text { for } i=1,2,3, \cdots, \\
C_{j, j}^{\prime} & =C_{j+1, j} \times C_{j+2, j} \times C_{j+3, j} \times \cdots \text { for } j=0,1,2 \cdots .
\end{aligned}
$$

Together with (5) and (6) the last three formulas yield

$$
\begin{aligned}
& B_{i}=B_{i, 0} \times B_{i, 1} \times B_{i, 2} \times \cdots, \\
& C_{j}=C_{0, j} \times C_{1, j} \times C_{2, j} \times \cdots .
\end{aligned}
$$

Thus the two original decompositions of $A$ have the refinements 


$$
A=\prod_{i<\infty} \prod_{j<\infty} B_{i, j}=\prod_{i<\infty} \prod_{j<\infty} C_{i, j},
$$

and according to (9) these are centrally isomorphic.

5. Decompositions into indecomposable factors. In order to prove the existence of centrally isomorphic refinements for two decompositions with countably many factors we had to assume that all the factors involved had the $\boldsymbol{H}_{0}$-exchange property. In proving that two decompositions with indecomposable factors are centrally isomorphic we can get by with a much weaker assumption. This is due to the next two lemmas.

Lemma 5.1. If an indecomposable algebra $B$ has the 2-exchange property, then $B$ has the exchange property.

Proof. Suppose

$$
A=B \times C=\prod_{i \in I} D_{i} .
$$

Since each element of $A$ is contained in the product of finitely many factors $D_{i}$, there exists a finite subset $J$ of $I$ such that

$$
B \cap \prod_{i \in J} D_{i} \neq\{0\} \text {. }
$$

Letting

$$
E=\prod_{i \in I-J} D_{i}
$$

we have

$$
A=\prod_{i \in J} D_{i} \times E
$$

By $3.11 B$ has the finite exchange property, and there therefore exist subalgebras $D_{i}^{\prime} \subseteq D_{i}(i \in J)$ and $E^{\prime} \subseteq E$ such that

$$
A=B \times \prod_{i \in J} D_{i}^{\prime} \times E^{\prime}
$$

By the modular law we can find subalgebras $D_{i}^{\prime \prime}$ with $D_{i}=D_{i}^{\prime} \times D_{i}^{\prime \prime}$ for $i \in J$, and $E^{\prime \prime}$ with $E=E^{\prime} \times E^{\prime \prime}$. By 2.20,

$$
B \cong \prod_{i \in J} D_{i}^{\prime \prime} \times E^{\prime \prime} .
$$

But as $B$ is indecomposable, only one of the factors in this last product can be different from $\{0\}$. This cannot be the factor $E^{\prime \prime}$, for then we would have $D_{i}^{\prime}=D_{i}$ for all $i \in j$, and the product in (2) could not exist because of (1). Thus $E^{\prime \prime}=\{0\}, E^{\prime}=E$, and letting $D_{i}^{\prime}=D_{i}$ for all 
$i \in I-J$ we have

$$
A=B \times \prod_{i \in I} D_{i}^{\prime}
$$

Lemma 5.2. If an algebra $A$ is a direct product of subalgebras all of which have the 2-exchange property, then every indecomposable factor of $A$ has the exchange property.

Proof. Suppose

$$
A=B \times C=\prod_{i \in I} D_{i}
$$

where $B$ is indecomposable and the algebras $D_{i}$ have the 2-exchange property. By 5.1 it suffices to show that $B$ has the 2-exchange property. As in the preceding proof, we choose a finite subset $J$ of $I$ with

$$
B \cap \prod_{i \in J} D_{i} \neq\{0\}
$$

By 3.10 the algebra

$$
E=\prod_{i \in J} D_{i}
$$

has the 2-exchange property, and there therefore exist subalgebras $B^{\prime} \subseteq B$ and $C^{\prime} \subseteq C$ such that

$$
A=E \times B^{\prime} \times C^{\prime} .
$$

By the modular law, $B^{\prime}$ is a factor of $B$, and because $B^{\prime} \cap E=\{0\} \neq$ $B \cap E$, we have $B^{\prime} \neq B$. Therefore $B^{\prime}=\{0\}$. Thus $A=B \times C=$ $E \times C^{\prime}$. Again by the modular law, $C=C^{\prime} \times\left(C \cap E^{\prime}\right)$, and using 2.20 we infer that $E \cong B \times\left(C \cap E^{\prime}\right)$. Thus $B$ is isomorphic to a factor of $E$, and therefore has the 2-exchange property by 3.10 .

THEOREM 5.3. If an algebra $A$ is a direct product of subalgebras all of which have the 2-exchange property, then any two direct decsmpositions of $A$ into indecomposable factors are centrally isomorphic.

Proof. Suppose

$$
A=\prod_{i \in I} B_{i}=\prod_{\jmath \in J} C_{j}
$$

where all the factors $B_{i}$ and $C_{j}$ are indecomposable and therefore, by 5.2, have the exchange property. For $I^{\prime} \cong I$ and $J^{\prime} \cong J$ let

$$
B\left(I^{\prime}\right)=\prod_{i \in I^{\prime}} B_{i} \text { and } C\left(J^{\prime}\right)=\prod_{j \in J^{\prime}} C_{j}
$$


and recall that, by $3.10, B\left(I^{\prime}\right)$ and $C\left(J^{\prime}\right)$ have the exchange property whenever the sets $I^{\prime}$ and $J^{\prime}$ are finite. In particular, it follows from this and the indecomposability of the factors $C_{j}$ that if $I^{\prime}$ is any finite subset of $I$, then $A=B\left(I^{\prime}\right) \times C\left(J-J^{\prime}\right)$ for some subset $J^{\prime}$ of $J$. Moreover, since $B\left(I^{\prime}\right) \cong{ }^{c} C\left(J^{\prime}\right)$, we see with the aid of 4.1 that $J^{\prime}$ must also be finite and that, in fact, there must exist a one-to-one map $\varphi$ of $I^{\prime}$ onto $J^{\prime}$ such that $B_{i} \cong{ }^{c} C_{\varphi(i)}$ for all $i \in I^{\prime}$. Similarly, for each finite subset $J^{\prime}$ of $J$ there exists a one-to-one map $\psi$ of $J^{\prime}$ into $I$ such that $C_{j} \cong{ }^{c} B_{\psi^{\prime}(j)}$ whenever $j \in J^{\prime}$.

For $k \in I$ let

$$
I_{k}=\left\{i \mid i \in I \text { and } B_{i} \cong{ }^{c} B_{k}\right\}, \quad J_{k}=\left\{j \mid j \in J \text { and } C_{j} \cong{ }^{c} B_{k}\right\} .
$$

From the above considerations we see that each member of $J$ must belong to at least one set $J_{k}$, and that if $J_{k}$ is finite, then $I_{k}$ must have at least as many elements as $J_{k}$. To complete the proof it suffices to show that this last statement also holds when $J_{k}$ is infinite. To prove this we consider, for each $i \in I$, the set $N_{i}$ of all elements $j \in J$ such that $A=B_{i} \times C(J-\{j\})$, and show that

$$
N_{i} \text { is finite for each } i \in I \text {, }
$$

$$
\bigcup_{i \in I_{k}} N_{i}=J_{k} \text {. }
$$

From this our assertion follows, for since $J_{k}$ is assumed to be infinite, (1) and (2) show that the number of elements in $J_{k}$ cannot exceed the number of distinct sets $N_{i}$ with $i \in I_{k}$, and hence cannot be larger than the number of elements in $I_{k}$.

Considering a fixed element $i \in I$, choose a finite subset $J^{\prime}$ of $J$ such that $B_{i} \cap C\left(J^{\prime}\right) \neq\{0\}$. Then the direct product $B_{i} \times C(J-\{j\})$ fails to exist whenever $j \in J-J^{\prime}$, and $N_{i}$ must therefore be a subset of $J^{\prime}$. Thus $N_{i}$ is finite.

Considering a fixed element $j \in J_{k}$, choose a finite subset $I^{\prime}$ of $I$ such that $C_{j} \cap B\left(I^{\prime}\right) \neq\{0\}$. Then there exists a finite subset $J^{\prime}$ of $J$ such that $A=B\left(I^{\prime}\right) \times C\left(J-J^{\prime}\right)$. Observing that $j \in J^{\prime}$, let $J^{\prime \prime}=$ $J^{\prime}-\{j$ ) and apply 3.9 to the direct decompositions

$$
A=C\left(J^{\prime \prime}\right) \times C_{j} \times C\left(J-J^{\prime}\right)=\prod_{i \in I^{\prime}} B_{i} \times C\left(J-J^{\prime}\right) .
$$

This yields and element $i \in I^{\prime}$ such that

$$
A=C\left(J^{\prime \prime}\right) \times B_{i} \times C\left(J-J^{\prime}\right)=B_{i} \times C(J-\{j\}),
$$

and therefore $j \in N_{i}$. Since $j \in J_{k}$ and $C_{j} \cong{ }^{c} B_{i}$, we have $i \in I_{k}$. Thus (2) holds, and the proof is complete. 
6. Factors with countably generated centers: Preliminary lemmas. As a result of Lemma 6.3 below the isomorphic refinement problem for algebras

$$
A=\prod_{i \in I} B_{i}
$$

where the factors $B_{i}$ have countably generated centers, reduces to the special case where $I$ is countable, and $A$ itself therefore has a countably generated center.

Lemma 6.1. If $B, C$ and $D_{i}(i \in I)$ are subalgebras of an algebra A such that

$$
A=B \times C=\prod_{i \in I} D_{i} \quad \text { and } \quad B^{c}=\prod_{i \in I}\left(B^{c} \cap D_{i}\right),
$$

then there exist subalgebras $E_{i}(i \in I)$ such that $B^{c} \cap D_{i} \subseteq E_{i} \subseteq D_{i}$ and

$$
A=C \times \prod_{i \in I} E_{i}
$$

Proof. By 2.16,

$$
A^{c}=B^{c} \times C^{c}=\prod_{i \in I} D_{i}^{c},
$$

and since each $B^{c} \cap D_{i}$ is a factor of $A^{c}$ and a subalgebra of $D_{i}^{c}$, it follows from the modular law that there exist subalgebras $D_{i}^{\prime}$ with $D_{i}^{c}=\left(B^{c} \cap D_{i}\right) \times D_{i}^{\prime}$ for all $i \in I$. Thus

$$
A^{c}=B^{c} \times C^{c}=B^{c} \times \prod_{i \in I} D_{i}^{\prime},
$$

and it follows from 2.19 and 2.18 that

$$
B \times C^{c}=B \times \prod_{i \in I} D_{i}^{\prime}=\prod_{i \in I} D_{i}^{\prime \prime}
$$

where $D_{i}^{\prime \prime}=\left(B \times C^{c}\right) \cap D_{i}$ for all $i \in I$. Again using the modular law we infer that, for each $i \in I, D_{i}^{\prime \prime}=D_{i}^{\prime} \times E_{i}$ where

$$
E_{i}=D_{i}^{\prime \prime} \cap\left(B \times \prod_{i \neq j \in I} D_{j}^{\prime}\right)
$$

Consequently

$$
B \times C^{c}=\prod_{i \in I} D_{i}^{\prime} \times \prod_{i \in I} E_{i}
$$

Observing that

$$
B^{c} \times C^{c}=B^{c} \times\left(\prod_{i \in I} D_{i}^{\prime}\right)^{c}=\left(\prod_{i \in I} E_{i}\right)^{c} \times\left(\prod_{i \in I} D_{i}^{\prime}\right)^{c},
$$


and that

$$
B^{c}=\prod_{i \in I}\left(B^{c} \cap D_{i}\right) \subseteq \prod_{i \in I} E_{i}^{c}=\left(\prod_{i \in I} E_{i}\right)^{c},
$$

we see with the aid of 2.4 that

$$
B^{c}=\left(\prod_{i \in I} E_{i}\right)^{c}
$$

Consequently

$$
C^{c} \cap\left(\prod_{i \in I} E_{i}\right)=\{0\}
$$

According to 2.14 this implies that the direct product

$$
A^{\prime}=C^{c} \times \prod_{i \in I} E_{i}
$$

exists. Furthermore, $A^{\prime}$ contains $B^{c} \times C^{c}$, and therefore contains all the algebras $D_{i}^{\prime}$. Hence it follows by (1) and (2) that $B \times C^{c} \leqq A^{\prime}$. The opposite inclusion also holds, since all the algebras $E_{i}$ are contained in $B \times C^{c}$. Thus $A^{\prime}=B \times C^{c}$. Together with (2) and 2.19 this yields the desired conclusion,

$$
A=B \times C=C \times \prod_{i \in I} E_{i} .
$$

Lemma 6.2. Suppose $B_{i}(i \in I), C_{j}(j \in J)$ and $D$ are subalgebras of an algebra $A$ such that

$$
A=\prod_{i \in I} B_{i} \times D=\prod_{j \in J} C_{j} \times D,
$$

and suppose $B_{i}^{c}$ is countably generated for each $i \in I$. If $k \in I$, then there exist a countable set $K \cong I$ with $k \in K$ and subalgebras $F_{j} \leqq C_{j}$ $(j \in J)$ such that

$$
\begin{aligned}
& A=\prod_{i \in I-K} B_{i} \times \prod_{j \in J} F_{j} \times D, \\
& \prod_{i \in K} B_{i}^{c} \times D^{c}=\prod_{j \in J} F_{j}^{c} \times D^{c} .
\end{aligned}
$$

Proof. Since $B_{k}^{c}$ is countably generated there exist countably generated subalgebras $E_{j, 0} \subseteq C_{j}^{c}(j \in J)$ such that $E_{j, 0}=\{0\}$ for all but countably many $j \in J$ and such that

$$
B_{k}^{c} \subseteq \prod_{j \in J} E_{j, 0} \times D^{c} .
$$

Since the algebra 


$$
F_{0}=\prod_{j \in J} E_{j, 0}
$$

is countably generated, there exists a countable subset $I_{1}$ of $I$ such that $k \in I_{1}$ and

$$
F_{0} \leqq \prod_{i \in I_{1}} B_{i}^{c} \times D^{c}
$$

Again, since the algebra

$$
G_{1}=\prod_{j \in I_{1}} B_{i}^{c}
$$

is countable generated, there exist countably generated subalgebras $E_{j, 1} \subseteq C_{j}^{c}(j \in J)$ such that $E_{j, 0} \subseteq E_{j, 1} \subseteq C_{j}$ for all $j \in J, E_{j, 1}=\{0\}$ for all but countably many $j \in J$, and

$$
G_{1} \subseteq \prod_{j \in J} E_{j, 1} \times D^{c}
$$

Continuing in this manner we obtain an ascending sequence of countable sets $I_{0}=\{k\} \subseteq I_{1} \subseteq I_{2} \subseteq \cdots \sqsubseteq I$ and, for each $j \in J$, an ascending sequence of subalgebras $E_{j, 0} \subseteq E_{j, 1} \subseteq E_{j, 2} \subseteq \cdots \subseteq C_{j}^{e}$ such that

$$
\prod_{i \in I_{n}} B_{i}^{c} \subseteq \prod_{j \in J} E_{j, n} \times D^{c} \cong \prod_{i \in I_{n+1}} B_{i}^{c} \times D^{c}
$$

for $n=0,1,2, \cdots$. Letting $K=I_{0} \cup I_{1} \cup \cdots$ and $E_{j}=E_{j, 0} \cup E_{j, 1} \cup \cdots$ for all $j \in J$ we therefore have

$$
\prod_{i \in K} B_{i}^{c} \times D^{c}=\prod_{j \in J} E_{j} \times D^{c} .
$$

Letting $\theta$ be the congruence relation over $A$ induced by $D$ we have

$$
A / \theta=\left(\left(\prod_{i \in I-K} B_{i}\right) / \theta\right) \times\left(\left(\prod_{i \in K} B_{i}\right) / \theta\right)=\prod_{j \in J}\left(C_{j} / \theta\right)
$$

by 3.3 and 3.7. Letting

$$
\bar{A}=\left(\left(\prod_{i \in \boldsymbol{K}} B_{i}\right) / \theta\right)^{c}
$$

we see by (1) that

$$
\bar{A}=\prod_{j \in J}\left(E_{j} / \theta\right),
$$

and it readily follows that $E_{j} / \theta=\bar{A} \cap\left(C_{j} / \theta\right)$ for all $j \in J$. We therefore infer by 6.1 that there exist subalgebras $F_{j}(j \in J)$ with $E_{j} \subseteq$ $F_{j} \subseteq C_{j}$ such that

$$
A / \theta=\left(\left(\prod_{i \in I-\underline{K}} B_{i}\right) / \theta\right) \times \prod_{j \in J}\left(F_{j} / \theta\right),
$$


and we conclude by 3.8 that (ii) holds. Finally, $\dot{E}_{j} \subseteq F_{j}^{c}$ for all $j \in J$, so that by (1)

$$
\prod_{i \in K} B_{i}^{c} \times D^{c} \cong \prod_{j \in J} F_{j}^{c} \times D^{c}
$$

Since, by (i) and (ii),

$$
A^{c}=\prod_{i \in I-K} B_{i}^{c} \times \prod_{i \in K} B_{i}^{c} \times D^{c}=\prod_{i \in I-K} B_{i}^{c} \times \prod_{j \in J} F_{i}^{c} \times D^{c},
$$

we conclude with the aid of 2.4 that (iii) holds.

LeMmA 6.3. If $B_{i}(i \in I)$ and $C_{j}(j \in J)$ are subalgebras of an algebra $A$ such that

$$
A=\prod_{i \in I} B_{i}=\prod_{j \in J} C_{j},
$$

and if $B_{i}^{c}$ is countably generated for each $i \in I$, then there exist a (possibly transfinite) sequence of countable pairwise disjoint subsets $I_{a}(\alpha<\lambda)$ of $I$ and subalgebras $C_{j, \alpha} \subseteq C_{j}(j \in J, \alpha<\lambda)$ of $A$ such that $I=\bigcup_{a<\lambda} I_{\infty}$ and, for all $\beta \leqq \lambda$,

$$
A=\prod_{\beta \leqq \alpha<\lambda} \prod_{i \in I_{\alpha}} B_{i} \times \prod_{j \in J} \prod_{\alpha<\beta} C_{j, \omega} .
$$

Proof. Letting $U_{\beta}=\mathrm{U}_{\alpha<\beta} I_{\alpha}$, we can write this last formula in the form

$$
A=\prod_{i \in I-U_{\beta}} B_{i} \times \prod_{j \in J} \prod_{\alpha<\beta} C_{j, \infty}
$$

Since this condition involves only sets $I_{\alpha}$ and algebras $C_{j, \alpha}$ with $\alpha<\beta$, it can be used as an induction hypothesis. To secure the convergence of our construction process we impose as a second induction hypothesis the condition

$$
\prod_{i \in U_{\beta}} B_{i}^{c} \subseteq \prod_{j \in J} \prod_{\alpha<\beta} C_{j, \infty}
$$

First observe that this last condition does in fact permit the passage through the limit ordinals. More precisely, suppose $\eta$ is a limit ordinal, and suppose the sets $I_{\alpha}$ and algebras $C_{j, \alpha}$ have been chosen for all $\alpha<\eta$ in such a way that (1) and (2) hold for all $\beta<\eta$. We wish to show that in this case (1) and (2) also hold for $\beta=\eta$. From the fact that the condition (2) holds for $\beta<\eta$ it follows that this condition also holds for $\beta=\eta$. Furthermore, since the direct product

$$
\prod_{i \in I-U \eta} B_{i} \times \prod_{j \in J} \prod_{\alpha<\beta} C_{j, \alpha}
$$

exists for all $\beta<\eta$, we readily see that the direct product

$$
A^{\prime}=\prod_{i \in I-U_{\eta}} B_{i} \times \prod_{j \notin J} \prod_{\alpha<\eta} C_{j, \alpha}
$$


also exists. In order to prove that $A^{\prime}=A$, and hence that (1) holds for $\beta=\eta$, it suffices to show that $B_{h} \subseteq A^{\prime}$ whenever $h \in U_{\eta}$. For each such index $h$ there exists an ordinal $\gamma<\eta$ with $h \in U_{\gamma}$. Using (1) with $\beta=\gamma$, (2) with $\beta=\eta$, and 2.19 we conclude that

$$
\begin{aligned}
& B_{h} \leqq \prod_{i \in I-U_{\gamma}} B_{i}^{c} \times \prod_{i \in U_{\gamma}} B_{i}=\prod_{i \in I-U_{\gamma}} B_{i}^{c} \times \prod_{j \in J} \prod_{\alpha<\gamma} C_{j, \alpha} \\
& \quad \subseteq \prod_{i \in I-U_{\eta}} B_{i}^{c} \times \prod_{j \in J} \prod_{\alpha<\eta} C_{j, \alpha} \subseteq A^{\prime} .
\end{aligned}
$$

Now consider an arbitrary ordinal $\eta$ and suppose the sets $I_{a b}$ and algebras $C_{j, \alpha} \leqq C_{j}(j \in J)$ have been defined for all $\alpha<\eta$ in such a way that (1) and (2) hold whenever $\beta \leqq \eta$. If $U_{\eta}=I$, then we take $\lambda=\eta$. Assuming that $U_{\eta} \neq I$, let

$$
\begin{aligned}
D_{j, \eta} & =\prod_{\alpha<\eta} C_{j, \alpha} \quad(j \in J), \\
D_{\eta} & =\prod_{j \in J} D_{j, \eta} .
\end{aligned}
$$

For each $j \in J, D_{j, \eta}$ is a factor of $A$ and a subalgebra of $C_{j}$, hence $C_{j}=D_{j, \eta} \times C_{j, \eta}^{\prime}$ for some subalgebra $C_{j \eta}^{\prime}$. It follows that

$$
A=\prod_{i \in I-U_{\eta}} B_{i} \times D_{\eta}=\prod_{j \in J} C_{j \eta}^{\prime} \times D_{\eta} .
$$

Choosing $k \in I-U_{\eta}$ we infer by 6.2 that there exist a countable set $I_{\eta}$ with $k \in I_{\eta} \subseteq I-U_{\eta}$ and subalgebras $C_{j, \eta} \subseteq C_{j \eta}^{\prime}(j \in J)$ such that

$$
\begin{gathered}
A=\prod_{i \in I-U_{\eta+1}} B_{i} \times \prod_{j \in J} C_{j, \eta} \times D_{\eta}=\prod_{i \in I-U_{\eta+1}} B_{i} \times \prod_{j \in J} \prod_{\alpha<\eta+1} C_{j, \infty}, \\
\prod_{i \in I_{\eta}} B_{i}^{c} \times D_{\eta}^{c}=\prod_{j \in J} C_{j, \eta}^{c} \times D_{\eta}^{c} .
\end{gathered}
$$

Here, in accordance with our earlier notation,

$$
U_{\eta+1}=\bigcup_{\alpha<\mu+1} I_{a}=U_{\eta} \cup I_{\eta} \text {. }
$$

By (3), (1) holds for $\beta=\eta+1$, and from (4) and the fact that (2) holds for $\beta=\eta$ we infer that (2) holds for $\beta=\eta+1$.

Since all the sets $I_{\alpha}$ are nonempty, there must exist an ordinal $\lambda$ such that $U_{\lambda}=I$, and the corresponding sets $I_{\alpha}$ and algebras $C_{j, \alpha}$ $(\alpha<\lambda, j \in J)$ clearly have the properties required by the lemma.

7. Factors with countably generated centers: Fundamental theorem. We are now ready to prove the fundamental theorem relating the exchange property to the isomorphic refinement property.

THEOREM 7.1. If an algebra $A$ is a direct product of subalgebras each of which has the exchange property and has a countably generated center, then any two direct decompositions of $A$ have centrally 
isomorphic refinements.

Proof. Suppose

$$
A=\prod_{i \in I} B_{i}
$$

where, for each $i \in I, B_{i}$ has the exchange property and $B_{i}^{c}$ is countably generated. Since every factor of $B_{i}$ (and hence every algebra isomorphic to such a factor) has the exchange property and has a countably generated center, it is enough to show that the decomposition (1) and any other decomposition

$$
A=\prod_{j \in J} C_{j}
$$

have centrally isomorphic refinements.

Consider first the case when $I$ is countable. For convenience suppose $I$ consists of the integers $0,1,2, \cdots$. In this case the center of $A$ is generated by a countable set

$$
Z=\left\{a_{0}, a_{1}, a_{2}, \cdots\right\} \text {. }
$$

We shall construct an increasing sequence of finite subsets $I_{0}, I_{1}, I_{2}, \ldots$ of $I$ and, for each $j \in J$, two sequences of subalgebras $D_{j, 0}, D_{j, 1}, D_{j, 2}, \ldots$ and $D_{j 0}^{\prime}=C_{j}, D_{j, 1}^{\prime}, D_{j, 3}^{\prime}, \cdots$ such that the following conditions hold for $k=0,1,2, \cdots$ :

$$
k \in I_{k}
$$

$$
D_{j, k}^{\prime}=D_{j, k} \times D_{j k+1}^{\prime} \quad \text { for all } j \in J \text {. }
$$

$$
A=\prod_{i \in I_{k}} B_{i} \times \prod_{i \in J} D_{j, k+1}^{\prime} \text {. }
$$

$$
a_{k} \in \prod_{j \in J} \prod_{l \leqq l} D_{j, l}
$$

By (2) there exists a finitely nonzero system of elements $c_{j, 0}^{\prime} \in C_{j}$ $(j \in J)$ such that

$$
a_{0}=\sum_{j \in J} c_{j, 0}^{\prime},
$$

and by (1) there exists a finite subset $I_{0}$ of $I$ such that $0 \in I_{0}$ and such that all the elements $c_{j 0}^{\prime}$ belong to the algebra

$$
B_{0}^{\prime}=\prod_{i \in I_{0}} B_{i}
$$

Since $B_{0}^{\prime}$ has the exchange property, there exist subalgebras $D_{j, 1}^{\prime} \subseteq C_{j}$ $(j \in J)$ such that (5) holds for $k=0$, and letting 


$$
D_{j, 0}=C_{j} \cap\left(B_{0}^{\prime} \times \prod_{j \neq h \in J} D_{h, 1}^{\prime}\right)
$$

for all $j \in \dot{J}$, we see that (4) and (6) also hold for $k=0$. In the case of (6) this is true because $c_{j, 0}^{\prime} \in C_{j} \cap B_{0}^{\prime} \subseteq D_{j, 0}$ for all $j \in J$.

Now consider an integer $n>0$, and assume that the finite subsets $I_{0} \subseteq I_{1} \subseteq \cdots \subseteq I_{n-1}$ of $I$ and the subalgebras $D_{j, 0}, D_{j, 1}, \cdots, D_{j, n-1}$, $D_{j, 0}^{\prime}=C_{j}, D_{j, 1}^{\prime}, \cdots, D_{j, n}^{\prime}(j \in J)$ have been so chosen that (3)-(6) hold for $k=0,1, \cdots, n-1$. For each $j \in J$ we have have

$$
C_{j}=\prod_{k<n} D_{j, k} \times C_{j, n}^{\prime},
$$

and there exist finitely non-zero systems of elements

$$
c_{j, n} \in \prod_{k<n} D_{j, k} \quad \text { and } \quad c_{j, n}^{\prime} \in D_{j, n}^{\prime} \quad(j \in J)
$$

such that

$$
a_{n}=\sum_{\jmath \in J} c_{j, n}+\sum_{j \in J} c_{j, n}^{\prime} .
$$

There exists a finite subset $I_{n}$ of $I$ such that $I_{n-1} \subseteq I_{n}$ and $n \in I_{n}$, and such that all the elements $c_{j, n}^{\prime}$ belong to the algebra

$$
B_{n}^{\prime}=\prod_{i \in J_{n}} B_{i} \text {. }
$$

Since $B_{n}^{\prime}$ has the exchange property, and since

$$
A=\prod_{i \in I_{n}} B_{i} \times \prod_{i \in I-I_{n}} B_{i}=\prod_{i \in I_{n-1}} B_{i} \times \prod_{j \in J} D_{j, n}^{\prime},
$$

there exist subalgebras $D_{j, n+1}^{\prime} \subseteq D_{j, n}^{\prime}(j \in J)$ such that (5) holds with $k=n$, and letting

$$
D_{j, n}=D_{j, n}^{\prime} \cap\left(B_{n}^{\prime} \times \prod_{j \neq h \in J} D_{h, n+1}^{\prime}\right)
$$

for all $j \in J$, we see that (4) and (6) also hold for $k=n$. In the case of (6) this is true because of the first formula in (7) and because of the fact that $c_{j, n}^{\prime} \in D_{j, n}^{\prime} \cap B_{n}^{\prime} \subseteq D_{j, n}$ for all $j \in J$. Thus we see that the sets $I_{k}$ and algebras $D_{j, k}$ and $D_{j, k}^{\prime}$ can be so chosen that (3)-(6) hold for $k=0,1,2, \cdots$.

It follows from (4) that the direct products

$$
C_{j}^{*}=\prod_{k<\infty} D_{j, k}(j \in J) \text { and } A^{*}=\prod_{j \in J} C_{j}^{*}
$$

exist, and from (6) we infer that $A^{c} \subseteq A^{*}$. Moreover, for any natural number $n$,

$$
A=\prod_{j \in J} \prod_{k \leqq n} D_{j, k} \times \prod_{j \in J} D_{j, n}^{\prime}
$$


and using this together with (3) and (5) we see by 2.19 that

$$
B_{n} \subseteq \prod_{i \in I_{n}} B_{i} \times\left(\prod_{j \in J} D_{j, n}^{\prime}\right)^{c}=\prod_{j \in J} \prod_{k \leqq n} D_{j, k}^{\prime} \times\left(\prod_{j \in J} D_{j, n}^{\prime}\right)^{c} \sqsubseteq A^{*} .
$$

Consequently $A^{*}=A$, and we infer by 2.4 that $C_{j}^{*}=C_{j}$ for all $j \in J$.

From (4) and (5) we see that

$$
\begin{aligned}
A & =\prod_{i \in I_{n-1}} B_{i} \times \prod_{j \in J} D_{j, n} \times \prod_{j \in J} D_{j, n+1}^{\prime} \\
& =\prod_{i \in I_{n-1}} B_{i} \times \prod_{i \in I_{n}-I_{n-1}} B_{i} \times \prod_{j \in J} D_{j, n+1}^{\prime},
\end{aligned}
$$

whence it follows that

$$
\prod_{i \in I_{n}-I_{n-1}} B_{i} \cong \prod_{j \in J}^{c} D_{j, n} \text {. }
$$

Consequently, by 4.1, there exist subalgebras $B_{i, j}$ and $C_{i, j},\left(i \in I_{n}-I_{n-1}\right.$, $j \in J$ ) such that

$$
\begin{aligned}
B_{i} & =\prod_{j \in J} B_{i, j} & & \text { for all } i \in I_{n}-I_{n-1}, \\
D_{j, n} & =\prod_{i \in I_{n}-I_{n-1}} C_{i, j} & & \text { for all } j \in J, \\
B_{i, j} & \cong{ }^{c} C_{i, j} & & \text { for all } i \in I_{n}-I_{n-1} \text { and } j \in J .
\end{aligned}
$$

Inasmuch as this holds for every natural number $n$ (with $I_{-1}=\varnothing$ ), we conclude that

$$
A=\prod_{i \in I} \prod_{j \in J} B_{i, j}=\prod_{i \in I} \prod_{j \in J} C_{i, j},
$$

and that these two decompositions of $A$ are centrally isomorphic and are refinements of the decompositions (1) and (2), respectively.

We now drop the assumption that $I$ is denumerable. By 6.3 there exist a sequence of countable, pairwise disjoint subsets $I_{\alpha}(\alpha<\lambda)$ of $I$, and for each $j \in J$ a sequence of subalgebras $D_{j, \alpha}(\alpha<\lambda)$ of $C_{j}$ such that $I=\mathrm{U}_{\alpha<\lambda} I_{\alpha}$ and

$$
A=\prod_{\beta \subseteq \alpha<\lambda} \prod_{i \in I_{\alpha}} B_{i} \times \prod_{j \in J} \prod_{\alpha<\beta} D_{j, \alpha}
$$

for all $\beta \leqq \lambda$. For $\beta=\lambda$ this yields

$$
A=\prod_{j \in J} \prod_{\alpha<\lambda} D_{j, \alpha},
$$

and using 2.4 we infer that

$$
C_{j}=\prod_{\alpha<\lambda} D_{j, \alpha} \quad \text { for all } j \in J .
$$

Taking in (8) two successive values for $\beta$, say $\beta=\gamma$ and $\beta=\gamma+1$, and comparing the resulting formulas, we see that 


$$
\prod_{I \in I_{\gamma}} B_{i} \cong{ }^{c} \prod_{j \in J} D_{j, \gamma} \text {. }
$$

Since $I_{\gamma}$ is countable, it follows from the first part of the proof that the two decompositions in (9) have centrally isomorphic refinements, and inasmuch as this holds for every $\gamma<\lambda$, we conclude that the decompositions (1) and (2) have centrally isomorphic refinements.

The preceding theorem can also be stated in the following, apparently more general, form.

THEOREM 7.2. If an algebra $A$ has two direct decompositions,

$$
A=\prod_{i \in I} B_{i}=\prod_{j \in J} C_{j},
$$

such that each of the factors $B_{i}(i \in I)$ has a countably generated center and each of the factors $C_{j}(j \in J)$ has the $\aleph_{0}$-exchange property, then any two direct decompositions of $A$ have centrally isomorphic refinemients.

Proof. Choosing the ordinal $\lambda$, subsets $I_{\alpha}(\alpha<\lambda)$ of $I$, and subalgebras $C_{j, \alpha}(j \in J, \alpha<\lambda)$ according to 6.3 , we have

$$
\begin{aligned}
C_{j} & =\prod_{\alpha<\lambda} C_{j, \alpha} & \text { for each } j \in J, \\
\prod_{i \in I_{\alpha}} B_{i} & \cong{ }^{c} \prod_{j \in J} C_{j, \alpha} & \text { for each } \alpha<\lambda .
\end{aligned}
$$

Since, by hypothesis, each of the sets $I_{a}$ is countable, the first direct product in (2) has a countably generated center, and hence so does the second product. Consequently each of the factors $C_{j, \infty}$ has a countably generated center. Furthermore, by (1) and 3.10, each of the algebras $C_{j, a}$ has the $\boldsymbol{\aleph}_{0}$-exchange property. Hence, by 3.12 , all the algebras $C_{j, \infty}$ have the exchange property. Since

$$
A=\prod_{j \in J} \prod_{\alpha<\lambda} C_{j, a},
$$

the conclusion now follows from 7.1.

8. Sufficient conditions for an algebra to have the $m$-exchange property. So far we have been primarily concerned with consequences of the exchange property, but in the remainder of this paper we shall investigate conditions that imply that a given algebra has the exchange property. In the present section it will be shown that this problem reduces to considerations that involve only abelian algebras.

THeOREM 8.1. For any cardinal $m$, if the center of an algebra $B$ has the m-exchange property, then $B$ has the m-exchange property. 
Proof. Suppose

$$
A=B \times C=\prod_{i \in I} D_{i},
$$

where the cardinal of $I$ is at most $m$. Then by 2.18 ,

$$
B^{c} \times C=\prod_{i \in I} D_{i}^{\prime}
$$

where $D_{i}^{\prime}=\left(B^{c} \times C\right) \cap D_{i}$ for each $i \in I$. Hence there exist subalgebras $E_{i} \subseteq D_{i}^{\prime}(i \in I)$ such that

$$
B^{c} \times C=B^{c} \times \prod_{i \in I} E_{i},
$$

and we conclude by 2.19 that

$$
A=B \times \prod_{i \in I} E_{i}
$$

THEOREM 8.2. For any cardinal $m$, in order for an algebra $B$ to have the m-exchange property it is sufficient (and obviously necessary) that the following condition be satisfied: For any algebra $A$ containing $B$ as a factor, and for any subalgebras $C$ and $D_{i}(i \in I)$ of $A$, if

$$
A=B \times C=\prod_{i \in I} D_{i},
$$

if the cardinal of $I$ does not exceed $m$, and if each of the algebras $D_{i}(i \in I)$ is isomorphic to a subalgebra of $B$, then there exist subalgebras $E_{i} \subseteq D_{i}(i \in I)$ such that

$$
A=B \times \prod_{i \in I} E_{i}
$$

Proof. Assume that the above condition is satisfied. Suppose

$$
A=B \times C=\prod_{i \in I} D_{i},
$$

where the cardinal of $I$ does not exceed $m$. Let $f$ and $g$ be the projections of $A$ onto $B$ and $C$ induced by the first decomposition of $A$, and for $i \in I$ let $h_{i}$ be the projection of $A$ onto $D_{i}$ induced by the second decomposition.

Let $\theta$ be the congruence relation over $A$ defined by the condition that, for all $x, y \in A$,

$$
x \theta y \text { if and only if } f h_{i}(x)=f h_{i}(y) \text { whenever } i \in I \text {. }
$$

We shall show that

(1) $\theta$ is consistent with the decompositions (i) of $A$. 
(2) The restriction of $\theta$ to $B$ is the identity relation over $B$.

Suppose $x, y \in A$ and $x \theta y$. Then

$$
\begin{aligned}
f(x) & =f\left(\sum_{i \in I} h_{i}(x)\right)=\sum_{i \in I} f h_{i}(x) \\
& =\sum_{i \in I} f h_{i}(y)=f\left(\sum_{i \in I} h_{i}(y)\right)=f(y) .
\end{aligned}
$$

In particular $f(x) \theta f(y)$. Moreover, this shows that for $x, y \in B$ the condition $x \theta y$ implies that $x=f(x)=f(y)=y$, so that (2) holds. Again assuming that $x \theta y$, if $k \in I$ then

$$
\begin{aligned}
& f h_{i} h_{k}(x)=0=f h_{i} h_{k}(y) \quad \text { whenever } k \neq i \in I, \\
& f h_{k} h_{k}(x)=f h_{k}(x)=f h_{k}(y)=f h_{k} h_{k}(y),
\end{aligned}
$$

so that $h_{k}(x) \theta h_{l}(y)$. From the equations

$$
\begin{aligned}
f h_{k} f(x)+f h_{k} g(x) & =f h_{k}(f(x)+g(x))=f h_{k}(x)=f h_{k}(y) \\
& =f h_{k}(f(y)+g(y))=f h_{k} f(y)+f h_{k} g(y)
\end{aligned}
$$

we infer that

$$
h_{i} f h_{k} f(x)+h_{i} f h_{k} g(x)=h_{i} f h_{k} f(y)+h_{i} f h_{k} g(y)
$$

for all $i, k \in I$. Since $f(x)=f(y)$, we have

$$
h_{i} f h_{k_{k}} f(x)=h_{i} f h_{k} f(y)
$$

for all $i, k \in I$, and if $i \neq k$, then this element belongs to $A^{c}$. Therefore, by (3),

$$
h_{i} f h_{k} g(x)=h_{i} f h_{k} g(y) \quad \text { whenever } i, k \in I \text { and } i \neq k \text {. }
$$

Considering now a fixed index $i \in I$, observe that

$$
\sum_{k \in I} h_{i} f h_{k} g(x)=h_{i} f\left(\sum_{k \in I} h_{k} g(x)\right)=h_{i} f g(x)=h_{i}(0)=0,
$$

with the corresponding formula holding with $x$ replaced by $y$. Hence, in particular,

$$
\sum_{k \in I} h_{i} f h_{k} g(x)=\sum_{k \in I} h_{i} f h_{k} g(y) .
$$

Furthermore, all the summands in these two sums belong to $A^{c}$ because $f h_{k} g(x)$ and $f h_{k} g(y)$ always belong to $A^{c}$. Since, by (4),

$$
\sum_{i \neq k \in I} h_{i} f h_{k} g(x)=\sum_{i \neq k \in I} h_{i} f h_{k} g(y),
$$

this implies that 


$$
h_{i} f h_{i} g(x)=h_{i} f h_{i} g(y) .
$$

Thus in (4) we can omit the condition that $i \neq k$, and we conclude that, for all $k \in I$,

$$
f h_{k} g(x)=\sum_{i \in I} h_{i} f h_{k} g(x)=\sum_{i \in I} h_{i} f h_{k} g(y)=f h_{k} g(y),
$$

so that $g(x) \theta g(y)$. This completes the proof of (1).

From (1) it follows that

$$
A / \theta=(B / \theta) \times(C / \theta)=\prod_{i \in I}\left(D_{i} / \theta\right) .
$$

Notice that if $k \in I$ and $x, y \in D_{k}$, then the conditions $x \theta y$ and $f(x)=$ $f(y)$ are equivalent, and therefore the mapping

$$
x / \theta \rightarrow f(x) \quad\left(x \in D_{k}\right)
$$

is an isomorphism of $D_{k} / \theta$ into $B$. Since $B \cong B / \theta$, it follows that there exist subalgebras $\bar{E}_{i} \subseteq D_{i} / \theta(i \in I)$ such that

$$
A / \theta=(B / \theta) \times \prod_{i \in I} \bar{E}_{i} .
$$

Consequently, by 3.4 there exist subalgebras $E_{i} \subseteq D_{i}(i \in I)$ such that

$$
A=B \times \prod_{i \in I} E_{i} .
$$

Because of 8.1 , we may apply the criterion in 8.2 to $B^{c}$ in place of $B$, and thus consider decompositions

$$
A=B^{\mathrm{c}} \times C=\prod_{i \in I} D_{i}
$$

where the algebras $D_{i}$ are isomorphic to subalgebras of $B^{c}$. However, the algebras $D_{i}$ need not be central subalgebras of $A$, and $A$ therefore is not necessarily abelian. We shall now show that it is actually sufficient to consider the case when $A$ is abelian, in which case the factors $C$ and $D_{i}(i \in I)$ of $A$ are of course also abelian.

THeOREM 8.3. For any cardinal $m$, in order for an algebra $B$ to have the m-exchange property it is sufficient that the following condition be satisfied: For any abelian algebra $A$ containing $B^{c}$ as a factor, and for any subalgebras $C$ and $D_{i}(i \in I)$ of $A$, if

$$
A=B^{c} \times C=\prod_{i \in I} D_{i},
$$

if the cardinal of $I$ does not exceed $m$, and if each of the algebras $D_{i}(i \in I)$ is isomorphic to a subalgebra of $B^{c}$, then there exist subalgebras $E_{i} \subseteq D_{i}(i \in I)$ such that 


$$
A=B^{c} \times \prod_{i \in I} E_{i}
$$

Proof. By 8.1 it suffices to show that $B^{c}$ has the $m$-exchange property, and by 8.2 it is therefore enough to show that the condition in our theorem implies the property obtained from it by deleting the word "abelian." Assume therefore that (i) holds, that the cardinal of $I$ does not exceed $m$, and that each of the algebras $D_{i}(i \in I)$ is isomorphic to a subalgebra of $B^{c}$. Under the operation + each of the algebras $D_{i}$ is therefore a commutative cancellation semigroup, and hence so is $A$. Consequently $A$ can be embedded in an Abelian group $\bar{A}$ in such a way that each element of $\bar{A}$ is the difference of two elements of $A$. This extension of $A$ is unique up to isomorphism. Furthermore, there is a unique way of extending the operations $F_{t}(t \in T)$ to $\bar{A}$ in such a way that the resulting algebra is abelian: If $a_{k}=a_{k}^{\prime}-a_{k}^{\prime \prime}$ with $a_{k}^{\prime}, a_{k}^{\prime \prime} \in A$ for $k=0,1, \cdots, \rho(t)-1$, then we let

$$
F_{t}\left(a_{0}, a_{1}, \cdots, a_{\rho(t)-1}\right)=F_{t}\left(a_{0}^{\prime}, a_{i}^{\prime}, \cdots, a_{\rho(t)-1}^{\prime}\right)-F_{t}\left(a_{0}^{\prime \prime}, a_{1}^{\prime \prime}, \cdots, a_{\rho(t)-1}^{\prime \prime}\right) .
$$

That this definition is unambiguous and actually does yield an abelian algebra is an easy consequence of the fact that the equation

$$
\begin{aligned}
F_{t}\left(x_{0}+\right. & \left.y_{0}, x_{1}+y_{1}, \cdots, x_{\rho(t)-1}+y_{\rho(t)+1}\right) \\
& =F_{t}\left(x_{0}, x_{1}, \cdots, x_{\rho(t)-1}\right)+F_{t}\left(y_{0}, y_{1}, \cdots, y_{\rho(t)-1}\right)
\end{aligned}
$$

holds whenever the elements $x_{k}, y_{k}(k=0,1, \cdots, \rho(t)-1)$ belong to $A$.

For any subalgebra $X$ of $A$ let $\bar{X}$ be the smallest abelian subalgebra of $\bar{A}$ that contains $X$. Then $\bar{X}$ consists of all elements of the form $x-x^{\prime}$ with $x, x^{\prime} \in X$. It is easy to check the condition

$$
A=\prod_{j \in J} X_{j}
$$

implies that

$$
\bar{A}=\prod_{j \in J} \bar{X}_{j}
$$

In particular, since $\bar{B}^{c}=B^{c}$,

$$
\bar{A}=B^{c} \times \bar{C}=\prod_{i \in I} \bar{D}_{i}
$$

For each $i \in I, D_{i}$ is isomorphic to a subalgebra of $B^{c}$, and the same is therefore true of $\bar{D}_{i}$. Hence, by hypothesis, there exist subalgebras $F_{i} \subseteq \bar{D}_{i}(i \in I)$ such that

$$
\bar{A}=B^{c} \times \prod_{i \in I} F_{i} .
$$

Given an element $a \in A$, there exist an element $b \in B^{c}$ and a finitely 
nonzero system of elements $f_{i} \in F_{i}(i \in I)$ such that

$$
a=b+\sum_{i \in I} f_{i} .
$$

Since $-b \in A$, the element

$$
a-b=\sum_{i \in I} f_{i}
$$

belongs to $A$, and there exists a finitely nonzero system of elements $d_{i} \in D_{i}(i \in I)$ such that

$$
a-b=\sum_{i \in I} d_{i}
$$

Inasmuch as $d_{i}, f_{i} \in \bar{D}_{i}$ for all $i \in I$, we infer that $d_{i}=f_{i} \in D_{i} \cap F_{i}$ for all $i \in I$, and therefore

$$
a=b+\sum_{i \in I} d_{i} \in B^{c} \times \prod_{i \in I}\left(D_{i} \cap F_{i}\right) .
$$

It is now easy to show that (ii) holds with $E_{i}=D_{i} \cap F_{i}$ for all $i \in I$.

9. Factors with central chain conditions. In this section we will show that algebras satisfying certain central chain conditions have the exchange property and have countably generated centers, and these results will be applied to obtain the principal isomorphic refinement theorem for general algebras. The chain conditions involved are made precise in the following two definitions.

Definition 9.1. An algebra $A$ is said to satisfy the minimal condition if every nonempty family of subtractive subalgebras of $A$ has a minimal member. Similarly, A satisfies the maximal condition if every nonempty family of subtractive subalgebras has a maximal member.

Definition 9.2. An algebra $A$ is said to satisfy the local maximal condition if every finitely generated subtractive subalgebra of $A$ satisfies the maximal condition.

It should be noted that the minimal and (local) maximal conditions as defined above involve only subtractive subalgebras of an algebra $A$. In particular, since the subtractive subalgebras of an operator group are precisely its admissible subgroups, for groups the minimal and maximal conditions as defined in $9: 1$ and 9.2 are just the usual grouptheoretic chain conditions.

The first theorem of this section makes use of the following lemma which is a consequence of the results of Baer [1]. 
Lemma 9.3. ([1]; Theorem D p. 96 and Theorem 3 p. 93) ${ }^{5}$ Let $G$ be an operator group which satisfies the minimal and local maximal conditions. If $G=B \times C=D \times E$ where $B$ is indecomposable, then there exist factors $D^{\prime} \subseteq D$ and $E^{\prime} \subseteq E$ such that $G=B \times D^{\prime} \times E^{\prime}$.

Suppose now that $A$ is an abelian algebra with auxiliary operations $F_{t}(t \in T)$. For each $t \in T$ and each $k<\rho(t)$ define the unary operation $F_{k, t}$ by

$$
F_{k, t}(a)=F_{t}(0, \cdots, 0, a, 0, \cdots, 0) \quad \text { for all } a \in A
$$

Since $A$ is abelian, it follows that for each $t \in T$ and elements $a_{0}, \cdots, a_{\rho(t)-1} \in A$ we have

$$
F_{t}\left(a_{0}, \cdots, a_{\rho(t)-1}\right)=\sum_{k<\rho(t)} F_{k, t}\left(a_{k}\right) .
$$

Consequently the (subtractive) subalgebras of $A$ and the direct decompositions of $A$ remain unchanged if we replace the operations $F_{t}(t \in T)$ by the operations $F_{k, t}(k<\rho(t), t \in T)$. Moreover, this new system so obtained is obviously an abelian operator group. Hence the following lemma is immediate by $\mathbf{9 . 3}$.

Lemma 9.4. If $A$ is an abelian algebra which satisfies the minimal condition and the local maximal condition, and if $A=$ $B \times C=D \times E$ where $B$ is indecomposable, then there exist factors $D^{\prime} \subseteq D$ and $E^{\prime} \subseteq E$ such that $A=B \times D^{\prime} \times E^{\prime}$.

THEOREM 9.5. If the center $B^{c}$ of an algebra $B$ satisfies the minimal condition and the local maximal condition, then $B$ has the exchange property.

Proof. By 8.1 we may assume that $B=B^{c}$. Since $B$ satisfies the minimal condition, it is a direct product of finitely many indecomposable subalgebras, and therefore by 3.10 and 5.1 it is sufficient to show that $B$ has the 2-exchange property.

Consider an abelian algebra $A$ containing $B$ as a subalgebra, and algebras $C, D_{0}$ and $D_{1}$ such that $D_{0}$ and $D_{1}$ are isomorphic to subalgebras of $B$ and such that $A=B \times C=D_{0} \times D_{1}$. Then both $D_{0}$ and $D_{1}$ satisfy the minimal and local maximal conditions, and it readily follows that the same is true of $A$. Therefore by 9.4 there exist subalgebras $E_{0} \subseteq D_{0}$ and $E_{1} \subseteq D_{1}$ such that $A=B \times E_{0} \times E_{1}$, and we conclude by 8.3 that $B$ has the exchange property.

${ }^{5}$ See also Specht [8], pp. 250, 259 and 260. 
In order to apply the preceding theorem in conjunction with 7.1, we must further show that under the given hypothesis $B^{c}$ is countably generated. This observation is based on the following lattice-theoretic lemma. The terminology and simple facts from lattice theory used below can be found in Birkhoff [3].

LEMMA 9.6. If $L$ is an upper continuous modular lattice, if every decreasing sequence of elements of $L$ is countable, and if every element of $L$ is a join of finite dimensional elements, then every element of $L$ is a join of countably many finite dimensional elements.

Proof. First consider an element $a \in L$ that is a join of atoms. Then there exists an independence sequence $p_{0}, p_{1}, \cdots, p_{\xi}, \cdots(\xi<\lambda)$ of atoms of $L$ such that

$$
a=\sum_{\xi<\lambda} p_{\xi}
$$

Since the elements

$$
\sum_{\eta \leqq \xi<\lambda} p_{\xi} \quad(\eta<\lambda)
$$

form a strictly decreasing sequence, $\lambda$ must be countable, and therefore $a$ is the join of countably many atoms.

Now consider an arbitrary element $a \in L$. For each $n=1,2, \cdots$ let $P_{n}$ be the set of all the elements $x \in L$ with $x \leqq a$ whose dimension does not exceed $n$, and let $a_{n}=\sum P_{n}$. Then

$$
a=\sum_{n<\infty} a_{n} \text {. }
$$

By the first part of the proof there is a countable set $Q_{1} \subseteq P_{1}$ such that $a_{1}=\sum Q_{1}$. Suppose $n>1$ and $x \in P_{n}$. Then either $x \leqq a_{n-1}$ or $x+a_{n-1}$ covers $a_{n-1}$, since each member of $P_{n}-P_{n-1}$ covers at least one member of $P_{n-1}$. Consequently $a_{n}$ is the join of atoms in the quotient sublattice $a / a_{n-1}$. Since the hypothesis of the lemma is satisfied with $L$ replaced by this sublattice, we again use the first part of the proof to infer that

$$
a_{n}=a_{n-1}+\sum Q_{n}
$$

where $Q_{n}$ is a countable subset of $P_{n}-P_{n-1}$. It follows that each $a_{n}$ is a join of countably many finite dimensional elements, and therefore $a$ is also a join of countably many such element.

CoROLlaRy 9.7. If $B$ is an abelian algebra that satisfies the minimal condition and the local maximal condition, then $B$ is countably generated. 
Proof. The lattice $L$ of all subtractive subalgebras of $B$ is modular and upper continuous, and, by hypothesis, every decreasing sequence of elements of $L$ is finite. Also, if $C$ is a finitely generated subtractive subalgebra of $B$, then the lattice $L(C)$ of all subtractive subalgebras of $C$ satisfies the double chain condition. Consequently $L(C)$ is finite dimensional, i.e., $C$ is a finite dimensional element of $L$. Since every subtractive subalgebra of $B$ is the lattice join of finitely generated subtractive subalgebras, $L$ satisfies the hypothesis of 9.6. Hence $B$ is the lattice join of countably many finite dimensional elements of $L$; equivalently, $B$ is generated by the set-union of countably many subtractive subalgebras $C$ such that $L(C)$ is finite dimensional. But if $L(C)$ is finite dimensional, then $C$ is clearly finitely generated. Thus it follows that $B$ is countably generated.

Combining 9.5, 9.7 and 7.1 we obtain our principal isomorphic refinement theorem for algebras with auxiliary operations.

Theorem 9.8. If an algebra $A$ has a direct decomposition

$$
A=\prod_{i \in I} B_{i}
$$

such that, for each $i \in I, B_{i}^{c}$ satisfies the minimal condition and the local maximal condition, then any two direct decompositions of $A$ have centrally isomorphic refinements.

10. Lemmas on abelian groups. When applied to algebras without auxiliary operations $F_{t}$, Theorem 9.8 can be stated in the following equivalent form: If a binary algebra $A$ is a direct product of subalgebras $B_{i}(i \in I)$ such that, for each $i \in I, B_{i}^{c}$ is a direct product of finitely many primary cyclic and quasi-cyclic groups, then any two direct decompositions of $A$ have centrally isomorphic refinements. For every abelian group satisfied the local maximal condition, and the condition imposed on the abelian groups $B_{i}^{c}$ above is equivalent to the assertion that they satisfy the minimal condition. In the next section we shall obtain a result that is considerably more general than the one stated above. Here we list a number of known results and prove five lemmas concerning abelian groups that will be used in the proof of this more general theorem.

If $G$ is an abelian group and $n$ is an integer, then the subgroups $n G$ and $G[n]$ are defined by

$$
\begin{aligned}
n G & =\{n x \mid x \in G\}, \\
G[n] & =\{x \mid x \in G \text { and } n x=0\} .
\end{aligned}
$$

As usual, we say that an abelian group $G$ is divisible if $n G=G$ for every integer $n \neq 0$, and we say that $G$ is of bounded order if there 
exists an integer $n \neq 0$ such that $n G=\{0\} . \quad$ An abelian group is said to be reduced if it has no nonzero divisible subgroup, and by the reduced part of an abelian group $G$ we mean the quotient group $G / D$ where $D$ is the maximal divisible subgroup of $G$. If $X$ is a subset of a group $G$, then $[X]$ denotes the subgroup of $G$ generated by $X$; in particular, if $x \in G$, then the cyclic subgroup of $G$ generated by $x$ is denoted by $[x]$.

Let $G$ be an abelian $p$-group ( $p$ some prime). By the height of an element $x \in G$ we mean the largest integer $r$ such that $x \in p^{r} G$, if a largest such integer $r$ exists, otherwise the height of $x$ is $\infty$. Thus height $x=\infty$ if $x \in p^{n} G$ for $n=1,2, \cdots$, and height $x=r<\infty$ if $x \in p^{r} G$ but $x \notin p^{r+1} G$. Obviously the zero element of $G$ has infinite height; if this is the only element in $G$ of infinite height, then we say that $G$ has no elements of infinite height. Thus $G$ has no elements of infinite height if and only if $\bigcap_{n<\infty} p^{n} G=\{0\}$.

If $G$ is an abelian $p$-group with no elements of infinite height, then a topology can be introduced in $G$ by taking as a neighborhood basis for 0 the subgroup $p^{n} G(n=1,2, \cdots)$. This topology is called the p-adic topology of $G$. $G$ can be completed in its $p$-adic topology, and the torsion subgroup $\widetilde{G}$ of the topological completion of $G$ is also an abelian $p$-group without elements of infinite height. ${ }^{6}$

An abelian $p$-group $G$ is said to be torsion-complete if $G$ has no elements of infinite height, and $G$ is equal to the torsion subgroup of the topological completion of $G, G=\widetilde{G}$. Alternatively, $G$ is torsioncomplete if and only if $G$ has no elements of infinite height, and every Cauchy sequence $\left\{x_{k}\right\}_{k<\infty}$ of $G$, for which the orders of the elements $x_{k}$ are bounded, converges to a limit in $G .^{7}$ For convenience we will call a Cauchy sequence $\left\{x_{k}\right\}_{k<\infty}$, for which the orders of the $x_{k}$ are bounded, a bounded Cauchy sequence.

An explicit representation of torsion-complete abelian $p$-groups can be given as follows. Let $U_{1}, U_{2}, U_{3}, \cdots$ be a sequence of $p$-groups such that $U_{n}$ is a direct product of cyclic groups of order $p^{n}$ for each $n=1,2, \cdots$. Let $\Gamma$ be the Cartesian product of the groups $U_{1}, U_{2}$, $U_{3}, \cdots$, that is, $\Gamma$ is the set of all functions $f$ defined on the positive integers such that $f(n) \in U_{n}$, with addition defined component-wise. Then the torsion subgroup of $\Gamma$ is torsion-complete. Conversely, if $G$ is a torsion-complete abelian $p$-group, then there exists a sequence of

${ }_{6}$ This is essentially given by Kaplansky [7], p. 50 .

${ }^{7}$ Fuchs [5], p. 114, calls these groups closed. However, we have adopted the terminology of Kaplansky-[7], p. 54, in order to remain consistent with topological terminology. Fuchs' definition of Cauchy sequence also differs somewhat from ours in that he requires a Cauchy sequence to be bounded and converge at a specified rate. Again we have followed Kaplansky [7] in using the usual topological concept of Cauchy Sequence. 
groups $U_{1}, U_{2}, U_{3}, \cdots$, where $U_{n}$ is a direct product of cyclic groups of order $p^{n}$ for each $n=1,2, \cdots$, such that $G$ is isomorphic to the torsion subgroup of the Cartesian product of $U_{1}, U_{2}, U_{3}, \ldots .8$ In particular, every primary abelian group of bounded order is torsioncomplete, and every countable torsion-complete primary abelian group is necessarily of bounded order.

By a pure subgroup of an abelian $p$-group $G$ we mean a subgroup $S$ of $G$ such that $S \cap p^{n} G=p^{n} S$ for all $n=1,2, \ldots$. It is easily seen that the $p$-adic topology of a pure subgroup of an abelian $p$-group $G$ with no elements of infinite height is the same as the topology induced by the $p$-adic topology of $G$. A subgroup $U$ of a $p$-group $G$ is called a basic subgroup if $U$ has the following properties:

(i) $U$ is a direct product of cyclic groups;

(ii) $U$ is a pure subgroup of $G$;

(iii) the quotient group $G / U$ is divisible. A subset $X \subseteq G$ is independent if the subgroup $[X]$ generated by $X$ is the direct product of the cyclic subgroups $[x]$ generated by the elements $x \in X$. If in addition, $[X]$ is a pure subgroup of $G$, then $X$ is called a pure independent subset.

The following ten lemmas are well known; proofs and references to the original sources can be found in Fuchs [5] as indicated in each case.

Lemma 10.1. ([5], p. 62) If a subgroup $S$ of an abelian group $G$ is divisible, then $S$ is a factor of $G$.

LEMma 10.2. ([5], p. 64) A divisible abelian group is a direct product of subgroups each of which is isomorphic to either the additive group of rationals or a primary quasi-cyclic group.

Lemma 10.3. ([5], p. 78) If $S$ is a subgroup of an abelian $p$ group $G$, and if every element of $S[p]$ has the same height in $S$ as it does in $G$, i.e., if $S[p] \cap p^{n} G=S[p] \cap p^{n} S(n=1,2, \cdots)$, then $S$ is a pure subgroup of $G$.

LEMma 10.4. ([5], p. 78) If $S$ is a pure subgroup of an abelian p-group $G$, and if $S[p]=G[p]$, then $S=G$.

Lemma 10.5. ([5], p. 97) A subgroup $U$ of a primary abelian group $G$ is a basic subgroup if and only if $U$ is generated by a maximal pure independent subset of $G$.

Lemma 10.6. ([5], pp. 98 and 104) A primary abelian group $G$

${ }^{8}$ Fuchs [5], p. 114. 
has at least one basic subgroup, and all the basic subgroups of G are isomorphic.

Lemma 10.7. ([5], p. 104) If a primary abelian group $G$ is of bounded order, then the only basic subgroup of $G$ is $G$ itself.

Lemma 10.8. ([5], pp. 98-99) Let $G$ be an abelian p-group, and suppose that a subgroup $U$ is a direct product $U=U_{1} \times U_{2} \times U_{3} \times \cdots$, where $U_{n}$ is a direct product of cyclic groups of order $p^{n}$ for each $n=1,2, \cdots$. Then the following conditions are equivalent:

(i) $U$ is a basic subgroup of $G$;

(ii) $G=U_{1} \times \cdots \times U_{n} \times\left[p^{n} G \cup \bigcup_{k>n} U_{k}\right]$ for each $n=1,2, \cdots$;

(iii) $U_{1} \times \cdots \times U_{n}$ is a maximal factor of $G$ of bounded order $p^{n}$ for each $n=1,2, \cdots$.

LEMma 10.9. ([5], p. 112) If $G$ is a primary abelian group with no elements of infinite height, then there exists a torsion-complete primary abelian group containing $G$ as a pure subgroup.

LEMMA 10.10. ([5], p. 117) If $S$ is a pure subgroup of a primary abelian group $G$, and if $S$ itself is torsion-complete, then $S$ is a factor of $G$.

Lemma 10.11. If $U=V \times W$ is a basic subgroup of an abelian p-group $G$, and if $V$ is of bounded order, then there is a subgroup $H$ of $G$ such that $G=V \times H$ and $W \subseteq H$.

Proof. Since $U$ is a direct product of cyclic $p$-groups, there is an integer $m$ such that

$$
V=V_{1} \times \cdots \times V_{m} \text { and } W=W_{1} \times \cdots \times W_{m} \times W_{m}^{\prime},
$$

where $V_{k}$ and $W_{k}$ are direct products of cyclic groups of order $p^{k}(k=$ $1, \cdots, m)$, and $W_{m}^{\prime}$ is a direct product of cyclic groups of orders greater than $p^{m}$. Then

$$
U=\left(V_{1} \times W_{1}\right) \times \cdots \times\left(V_{m} \times W_{m}\right) \times W_{m}^{\prime},
$$

and hence by 10.8 ,

$$
G=V \times W_{1} \times \cdots \times W_{m} \times\left[p^{m} G \cup W_{m}^{\prime}\right] .
$$

Consequently the subgroup $H=\left[p^{m} G \cup W\right]$ has the required properties.

LEMma 10.12. If $X$ is a maximal pure independent subset of an abelian p-group $G$, and if $Y$ is a pure independent subset of $G$, then 
there exists a maximal pure independent subset $Z$ of $G$ such that $Y \subseteq Z \subseteq X \cup Y$.

Proof. By Zorn's Lemma there exists a pure independent subset $Z$ of $G$ which is maximal with respect to the property $Y \subseteq Z \subseteq X \cup Y$. Suppose $Z$ is not a maximal pure independent subset of $G$. Then there exists a maximal pure independent subset $Z^{\prime}$ such that $Z \subset Z^{\prime}$. Choose any $d \in Z^{\prime}-Z$. If the order of $d$ is $p^{n}$, let

$$
X_{n}=\left\{x \mid x \in X \text { and } p^{n} x=0\right\} .
$$

By 10.5 and 10.11 there exist subgroups $H_{0}$ and $H_{1}$ of $G$ such that $Z \subseteq H_{0}$ and

$$
G=[d] \times H_{0}=H_{1} \times \prod_{x \in X_{n}}[x] .
$$

Then there exist an element $e \in H_{1}$ and a finite subset $\left\{x_{0}, \cdots, x_{m-1}\right\} \subseteq$ $X_{n}$ such that

$$
d \in[e] \times\left[x_{0}\right] \times \cdots \times\left[x_{m-1}\right] .
$$

Observe that if $u \in G$ is an element of order at most $p^{n}$ such that $p^{n-1} u \notin H_{0}$, then $u$ has order exactly $p^{n}$, and $[u] \cap H_{0}=\{0\}$; therefore, as $H_{0}$ has index $p^{n}$ in $G$, we must have $G=[u] \times H_{0}$. Consequently, since $H_{1}$ contains no factor of order $p^{n}$ by 10.8 (iii), it follows that $p^{n-1} e \in H_{0}$. On the other hand, since $p^{n-1} d \notin H_{0}$, there exists $k<m$ such that $p^{n-1} x_{k} \notin H_{0}$. But then $G=\left[x_{k}\right] \times H_{0}$, and this implies that $Z \cup\left\{x_{k}\right\}$ is a pure subset of $G$ with $Y \subseteq Z \subset Z \cup\left\{x_{k}\right\} \subseteq X \cup Y$. Since this contradicts the choice of $Z$, it follows that $Z$ is a maximal pure independent subset of $G$.

Consider now a torsion-complete primary abelian group $G$ and a pure subgroup $S$ of $G$. Define $\bar{S}$ to be the subgroup consisting of all those elements $x \in G$ which are limits in $G$ of bounded Cauchy sequences of $S$. It is easy to see, and is implicit in the proof of the next lemma, that $S$ is just the topological closure of $S$ in $G$. Moreover, if $T$ is a pure torsion-complete subgroup of $G$ containing $S$, then $T \supseteqq \bar{S}$; in particular if $S$ itself is torsion-complete, then $\bar{S}=S$.

Lemma 10.13. If $S$ is a pure subgroup of a torsion-complete abelian p-group $G$, then $\bar{S}$ is a pure torsion-complete subgroup of $G$.

Proof. First observe that if $\left\{s_{k}\right\}_{k<\infty}$ is a Cauchy sequence of $S$ converging to an element $x$, and if $p^{m} x=0$, then there is a bounded Cauchy sequence $\left\{t_{k}\right\}_{k<\infty}$ of $S$ which converges to $x$ such that $p^{m} t_{k}=0$ for all $k$. By picking an appropriate subsequence, if necessary, we may assume that 


$$
x-s_{k} \in p^{k} G \quad(k=1,2, \cdots) .
$$

Since $p^{m} x=0$, we have $p^{m} s_{k} \in p^{k+m} G$. Thus, since $S$ is pure, there is an element $s_{k}^{\prime} \in S$ such that $p^{m} s_{k}=p^{k+m} s_{k}^{\prime}$ for each $k=1,2, \cdots$. Let $t_{k}=s_{k}-p^{k} s_{k}^{\prime}(k=1,2, \cdots)$. Then clearly $p^{m} t_{k}=0$, and

$$
x-t_{k}=\left(x-s_{k}\right)+p^{k} s_{k}^{\prime} \in p^{k} G \quad(k=1,2, \cdots),
$$

i.e., $\left\{t_{k}\right\}_{k<\infty}$ is a bounded Cauchy sequence of $S$, bounded by $p^{m}$, which converges to $x$.

Let $\left\{x_{k}\right\}_{k<\infty}$ be a bounded Cauchy sequence of $\bar{S}$. Since $G$ is torsion-complete, there is an element $x \in G$ which is the limit of $\left\{x_{k}\right\}_{k<\infty}$ in $G$. By picking an appropriate subsequence, if necessary, we may assume that

$$
x-x_{k} \in p^{k} G
$$$$
(k=1,2, \cdots) \text {. }
$$

Since $\left\{x_{k}\right\}_{k<\infty}$ is bounded, there is an integer $m$ such that $p^{m} x_{k}=0$ $(k=1,2, \cdots)$. Moreover, since each $x_{k} \in S$, there are Cauchy sequences $\left\{s_{k, n}\right\}_{n<\infty}$ such that $\left\{s_{k, n}\right\}_{n<\infty}$ converges to $x_{k}$ for each $k=1,2, \cdots$. And, as observed above, we can choose the $s_{k, n}$ such that

$$
p^{m} s_{k, n}=0 \quad \text { and } \quad x_{k}-s_{k, n} \in p^{n} G
$$

for all $n, k=1,2, \cdots$. Let $t_{k}=s_{k, k}$. Then

$$
x-t_{k}=\left(x-x_{k}\right)+\left(x_{k}-s_{k, k}\right) \in p^{k} G,
$$

and hence $\left\{t_{k}\right\}_{k<\infty}$ is a bounded Cauchy sequence of $S$ which converges to $x$. Therefore $x \in \bar{S}$, and $\bar{S}$ is torsion-complete.

To see that $\bar{S}$ is pure, let $x \in \bar{S}$, and suppose that $x \in p^{r} G$. Then there is a bounded Cauchy sequence $\left\{s_{k}\right\}_{k<\infty}$ of $S$ such that

$$
x-s_{k} \in p^{k} G
$$

and hence that

$$
s_{k+1}-s_{k} \in p^{k} G
$$

for all $k=1,2, \cdots$. Consequently $s_{r+1} \in p^{r} G$, and therefore, since $S$ is pure, there exist elements $t_{1} \in S$ and $s_{k}^{\prime} \in S(k=1,2, \cdots)$ such that

$$
p^{r} t_{1}=s_{r+1}, \text { and } s_{k+1}-s_{k}=p^{k} s_{k}^{\prime} \quad \text { for all } k=1,2, \cdots \text {. }
$$

Define elements $t_{k} \in S(k=1,2, \cdots)$ recursively by $t_{k+1}=t_{k}+p^{k} s_{r+k}^{\prime}$. Then clearly $\left\{t_{k}\right\}_{k<\infty}$ is a bounded Cauchy sequence of $S$ which converges to a limit $t \in \bar{S}$. Moreover, if $p^{r} t_{k}=s_{r+k}$, then

$$
p^{r} t_{k+1}=p^{r} t_{k}+p^{r+k} s_{r+k}^{\prime}=s_{r+k}+\left(s_{r+k+1}-s_{r+k}\right)=s_{r+k+1} ;
$$

hence $p^{r} t_{k}=s_{r+k}$ for all $k=1,2, \cdots$. It follows that $p^{r} t=x$, whence 
$x \in p^{r} \bar{S}$. Thus $\bar{S}$ is a pure subgroup of $G$.

COROLlaRY 10.14. If $U$ is a basic subgroup of a torsion-complete primary abelian group $G$, then $\bar{U}=G$.

Proof. By 10.10, 10.8 and 10.13.

LeMma 10.15. If $R=S \times T$ is a pure subgroup of a torsioncomplete abelian p-group $G$, then $\bar{R}=\bar{S} \times \bar{T}$.

Proof. Suppose $x \in \bar{S} \cap \bar{T}$ and $x \neq 0$. Then there are bounded Cauchy sequences $\left\{s_{k}\right\}_{k<\infty}$ and $\left\{t_{k}\right\}_{k<\infty}$ of $S$ and $T$, respectively, such that

$$
x-s_{k}, x-t_{k} \in p^{k} G \quad(k=1,2, \cdots) .
$$

Since $x \neq 0, x$ has height $r$ for some integer $r$. It follows that $s_{k}$ and $t_{k}$ must also have height $r$ for each $k>r$. And, as $s_{k} \in S, t_{k} \in T$, and $R=S \times T$ is a pure subgroup of $G$, it readily follows that $s_{k}-t_{k}$ has height $r$ for each $k>r$. But this is a contradiction since

$$
s_{k}-t_{k}=\left(x-t_{k}\right)-\left(x-s_{k}\right) \in p^{k} G \quad(k>r) .
$$

Consequently $\bar{S} \cap \bar{T}=\{0\}$. On the other hand, if $\left\{x_{k}\right\}_{k<\infty}$ is a bounded Cauchy sequence of $R$ converging to a limit $x \in \bar{R}$, then $\left\{f\left(x_{k}\right)\right\}_{k<\infty}$ and $\left\{g\left(x_{k}\right)\right\}_{k<\infty}$ are bounded Cauchy sequences of $S$ and $T$, respectively, where $f$ is the projection of $R$ onto $S$, and $g$ is the projection of $R$ onto $T$. Hence there are elements $u \in \bar{S}$ and $v \in \bar{T}$ which are the limits of $\left\{f\left(x_{k}\right)\right\}_{k<\infty}$ and $\left\{g\left(x_{k}\right)\right\}_{k<\infty}$, respectively. Since

$$
x_{k}=f\left(x_{k}\right)+g\left(x_{k}\right) \quad \text { for each } k=1,2, \cdots,
$$

it follows that $x=u+v$, and we conclude that $\bar{R}=\bar{S} \times \bar{T}$.

11. Exchange and isomorphic refinement theorems for binary algebras. In the present section conditions are found in order for a binary algebra $B$ to have the exchange property, and these conditions are combined with the results of preceeding sections to obtain uniqueness and isomorphic refinement theorems for binary algebras.

The center $B^{c}$ of a binary algebra $B$ can be written as a direct product

$$
B^{c}=P \times Q \times R
$$

where $P$ is a divisible torsion-free abelian group, $Q$ is a divisible torsion abelian group, and $R$ is a reduced abelian group. The groups $Q$ and 
$P \times Q$ are unique, and $P \times Q$ is the maximal divisible subgroup of $B^{c}$. Therefore $R$ is isomorphic to the reduced part of $B^{c}$. By 3.10, $B^{c}$ has the exchange property if and only if each of the factors $P, Q$ and $R$ has this property. In the case of $P$ the exchange property readily follows from 8.3 and some elementary properties of vector spaces. Since a torsion abelian group is uniquely a direct product of its primary components, it is clear that a torsion abelian group has the exchange property if and only if each of its primary components has the exchange property. In the case of divisible primary groups, and hence for $Q$, the exchange property again follows essentially from vector space properties. As for reduced groups, the main lemma of this section asserts that a torsion-complete primary abelian group has the exchange property. Consequently every torsion abelian group with torsion-complete primary components has the exchange property.

LEMMA 11.1. Every torsion-free divisible abelian group $G$ has the exchange property.

Proof. Using the criterion of 8.3, suppose

$$
A=G \times C=\prod_{i \in I} D_{i}
$$

where each of the factors $D_{i}(i \in I)$ is isomorphic to a subgroup of $G$. If $A^{\prime}, C^{\prime}$ and $D_{i}^{\prime}(i \in I)$ are the maximal divisible subgroups of $A, C$ and $D_{i}(i \in I)$, respectively, then

$$
A^{\prime}=G \times C^{\prime}=\prod_{i \in I} D_{i}^{\prime}
$$

Furthermore, for each $i \in I$ there is a subgroup $D_{i}^{\prime \prime}$ such that $D_{i}=$ $D_{i}^{\prime} \times D_{i}^{\prime \prime}$, and thus

$$
A=A^{\prime} \times \prod_{i \in I} D_{i}^{\prime \prime}
$$

Regarding $A^{\prime}$ as a vector space over the field of rational numbers, we can choose a basis $X$ for $G$ and extend it to a basis $Y$ for $A^{\prime}$ in such a way that every element of $Y-X$ belongs to one of the factors $D_{i}^{\prime}$. Letting $E_{i}^{\prime}$ be the vector space spanned by $D_{i}^{\prime} \cap(Y-X)$, we conclude that

$$
A^{\prime}=G \times \prod_{i \in I} E_{i}^{\prime}
$$

Therefore

$$
A=G \times \prod_{i \in I} E_{i}
$$

where $E_{i}=E_{i}^{\prime} \times D_{i}^{\prime \prime}(i \in I)$, and hence $G$ has the exchange property. 
Lemma 11.2. Every primary abelian group $G$ of bounded order has the exchange property.

Proof. Suppose

$$
A=G \times C=\prod_{i \in I} D_{i}
$$

where each of the factors $D_{i}$ is isomorphic to a subgroup of $G$. Then $A$ is a primary abelian group of bounded order. Let $Y$ be a maximal pure independent subset of $G$, and for each $i \in I$ let $X_{i}$ be a maximal pure independent subset of $D_{i}$. Then $X=\bigcup_{i \in I} X_{i}$ is a maximal pure independent subset of $A$, and it follows by 10.12 that there exists a maximal pure independent subset $Z$ of $A$ such that $Y \subseteq Z \subseteq X \cup Y$. By 10.5 and 10.7, $A$ is generated by $Z$, and $G$ is generated by $Y$. Consequently, if $E_{i}$ is the subgroup generated by the set $D_{i} \cap(Y-X)$ for each $i \in I$, it follows that

$$
A=G \times \prod_{i \in I} E_{i}
$$

Thus $G$ has the exchange property.

Lemma 11.3. Every divisible abelian p-group $G$ has the exchange property.

Proof. Suppose

$$
A=G \times C=\prod_{i \in I} D_{i}
$$

where each $D_{i}$ is isomorphic to a subgroup of $G$. If $A^{\prime}, C^{\prime}$ and $D_{i}^{\prime}(i \in I)$ are the maximal divisible subgroups of $A, C$ and $D_{i}(i \in I)$, respectively, then

$$
A^{\prime}=G \times C^{\prime} \times \prod_{i \in I} D_{i}^{\prime}
$$

Furthermore, if $D_{i}^{\prime \prime}$ is such that $D_{i}=D_{i}^{\prime} \times D_{i}^{\prime \prime}$ for each $i \in I$, then

$$
A=A^{\prime} \times \prod_{i \in I} D_{i}^{\prime \prime} \text {. }
$$

Clearly

$$
A^{\prime}[p]=G[p] \times C^{\prime}[p]=\prod_{i \in I} D_{i}^{\prime}[p],
$$

and since $G[p]$ is of bounded order $p$, there exist subgroups $U_{i} \subseteq$ $D_{i}^{\prime}[p](i \in I)$ such that

$$
A^{\prime}[p]=G[p] \times \prod_{i \in I} U_{i}
$$


For each $i \in I$ there exists a divisible subgroup $E_{i}^{\prime}$ of $D_{i}^{\prime}$ such that $E_{i}^{\prime}[p]=U_{i}$, and it follows from (1) that the direct product

$$
A^{\prime \prime}=G \times \prod_{i \in I} E_{i}^{\prime}
$$

exists. Moreover, since $A^{\prime \prime}$ is divisible, it is a pure subgroup of $A^{\prime}$, and using the fact that $A^{\prime}[p] \subseteq A^{\prime \prime}$ we infer by 10.4 that $A^{\prime}=A^{\prime \prime}$. Thus

$$
A=G \times \prod_{i \in I} E_{i}
$$

where $E_{i}=E_{i}^{\prime} \times D_{i}^{\prime \prime}$, and $G$ has the exchange property.

LEMMA 11.4. Every torsion-complete abelian p-group $G$ has the exchange property.

Proof. We first prove that $G$ has the 2-exchange property and hence the finite exchange property. Thus suppose

$$
A=G \times C=D_{0} \times D_{1}
$$

where $D_{0}$ and $D_{1}$ are isomorphic to subgroups of $G$. Then $A$ is an abelian $p$-group without elements of infinite height, and hence by 10.9 there is a torsion-complete abelian $p$-group $A^{\prime}$ containing $A$ as a pure subgroup. By 10.13 we may assume that $A^{\prime}$ is the closure of $A, A^{\prime}=\bar{A}$, and in this case it follows by 10.15 that

$$
\bar{A}=G \times \bar{C}=\bar{D}_{0} \times \bar{D}_{1} .
$$

Choose maximal pure independent subsets $X_{0}, X_{1}$ and $Y$ of $\bar{D}_{0}, \bar{D}_{1}$ and $G$ respectively. Then $X=X_{0} \cup X_{1}$ is a maximal pure independent subset of $\bar{A}$, and by 10.12 there is a maximal pure independent subset $Z$ of $A$ such that $Y \subseteq Z \subseteq X \cup Y$. Since every subset of $Z$ generates a factor of $[Z]$, the subgroups generated by $\bar{D}_{0} \cap Z$ and $\bar{D}_{1} \cap Z$ are pure in $\bar{A}$. Let $E_{0}$ and $E_{1}$ be the closures of the subgroups generated by $\bar{D}_{0} \cap Z$ and $\bar{D}_{1} \cap Z$, respectively. Then by 10.14 and 10.15 ,

$$
\bar{A}=G \times E_{0} \times E_{1} \text {. }
$$

Since $E_{0} \times\left(G \times E_{1}\right) \supseteqq D_{0} \times \bar{D}_{1} \supseteqq G \times E_{1}$, we infer from the modular law that

$$
D_{0} \times \bar{D}_{1}=\left(E_{0} \cap\left(D_{0} \times \bar{D}_{1}\right)\right) \times G \times E_{1}=G \times\left(D_{0} \cap E_{0}\right) \times E_{1} .
$$

Therefore $E_{1} \times\left(G \times\left(D_{0} \cap E_{0}\right)\right) \supseteqq D_{0} \times D_{1} \supseteqq G \times\left(D_{0} \cap E_{0}\right)$, and a second application of the modular law yields 


$$
\begin{aligned}
A=D_{0} \times D_{1} & =\left(E_{1} \cap\left(D_{0} \times D_{1}\right)\right) \times G \times\left(D_{0} \cap E_{0}\right) \\
& =G \times\left(D_{0} \cap E_{0}\right) \times\left(D_{1} \cap E_{1}\right) .
\end{aligned}
$$

Consequently $G$ has the 2-exchange property.

Now suppose

$$
A=G \times C=\prod_{i \in I} D_{i}
$$

where each of the factors $D_{i}$ is isomorphic to a subgroup of $G . \quad A$ is therefore an abelian $p$-group with no elements of infinite height. For each $i \in I$ let $f_{i}$ be the project of $A$ onto $D_{i}$ induced by the second decomposition in (1). We begin by proving the following statement:

(S) There exist a finite set $J \subseteq I$ and subgroups $G_{0}$ and $G_{1}$ such that $G=G_{0} \times G_{1}, G_{0}$ is of bounded order, and

$$
G_{1}[p] \subseteq \prod_{i \in J} D_{i}
$$

Assume that (S) is false. Then for every finite subset $J \subseteq I$ and every decomposition $G=G_{0} \times G_{1}$ where $G_{0}$ is of bounded order, there is an element $x \in G_{1}[p]$ and an index $i \in I-J$ such that $f_{i}(x) \neq 0$. Using this we shall construct a sequence of elements $x_{0}, x_{1}, x_{2}, \cdots \in G[p]$ and a sequence of indices $i_{1}, i_{2}, i_{3}, \cdots \in I$ such that the following conditions hold for every positive integer $n$ :

(3) height $x_{n}>$ height $f_{i}\left(x_{n-1}\right)$ whenever $i \in I$ and $f_{i}\left(x_{n-1}\right) \neq 0$;

$$
f_{i_{n}}\left(x_{0}\right)=f_{i_{n}}\left(\bar{x}_{1}\right)=\cdots=f_{i_{n}}\left(x_{n-1}\right)=0 \neq f_{i_{n}}\left(x_{n}\right) \text {. }
$$

Pick any element $x_{0} \in G[p]$. Suppose the elements $x_{1}, \cdots, x_{m} \in G[p]$ and the indices $i_{1}, \cdots, i_{m} \in I$ have been so chosen that (3) and (4) hold for $n=1, \cdots, m$. Then the set

$$
J_{m}=\left\{i \mid i \in I \text { and } f_{i}\left(x_{n}\right) \neq 0 \text { for some } n \leqq m\right\}
$$

is finite, and we can choose a positive integer $r$ such that

$$
r \geqq \text { height } f_{i}\left(x_{n}\right) \text { whenever } i \in J_{m}, n \leqq m \text { and } f_{i}\left(x_{n}\right) \neq 0 \text {. }
$$

By 10.8, $G$ has a decomposition $G=G_{0} \times G_{1}$ such that $p^{r+1} G_{0}=\{0\}$ and such that $G_{1}$ has no factor of order less that $p^{r+2}$. Therefore there exists and element $x_{m+1} \in G_{1}[p]$ and an index $i_{m+1} \in I-J_{m}$ such that $f_{i_{m+1}}\left(x_{m+1}\right) \neq 0$. Since the height of $x_{m+1}$ is necessarily larger than $r$, we infer from the choice of $r$ that (3) holds for $n=m+1$. Also, since $i_{m+1} \notin J_{m}$, it follows that (4) also holds with $n=m+1$. Thus the existence of the sequences of elements $x_{n} \in G[p]$ and of indices $i_{n} \in I$ satisfying (3) and (4) follows by induction.

For each $m=0,1,2, \cdots$ let 


$$
y_{m}=x_{0}+\cdots+x_{m} .
$$

If $m>n$, then it follows from (4) that

$$
f_{i_{n}}\left(y_{m}\right)=f_{i_{n}}\left(x_{n}\right)+\cdots+f_{i_{n}}\left(x_{m}\right) .
$$

From (3) we infer that the height of $f_{i_{n}}\left(x_{n}\right)$ is less than the height of $f_{i_{n}}\left(x_{k}\right)$ for $k=n+1, \cdots, m$. Consequently

$$
\text { height } f_{i_{n}}\left(y_{m}\right)=\text { height } f_{i_{n}}\left(x_{n}\right) \text { whenever } m>n \text {. }
$$

Notice that (3) also implies that the height of $x_{m}$ is at least $m$. Therefore

$$
y_{m+1}-y_{m}=x_{m+1} \in p^{m} G \quad(m=0,1,2, \cdots),
$$

and since each $y_{m}$ has order $p$, the sequence $\left\{y_{m}\right\}_{m<\infty}$ is a bounded Cauchy sequence of $G$ which must converge to a limit $y \in G$. Furthermore, for each $i \in I$, the sequence $\left\{f_{i}\left(y_{m}\right)\right\}_{m<\infty}$ is a bounded Cauchy sequence of $D_{i}$ which converges to $f_{i}(y)$. Now $f_{i}(y)=0$ for all but finitely many $i \in I$, and therefore there is a positive integer $n$ such that $f_{i_{n}}(y)=0$. But the sequence $\left\{f_{i_{n}}\left(y_{m}\right)\right\}_{m<\infty}$ cannot converge to 0 , since according to (5) the heights of the elements $f_{i_{n}}\left(y_{0}\right), f_{i_{n}}\left(y_{1}\right)$, $f_{i_{n}}\left(y_{2}\right), \cdots$ are bounded. Thus we have a contradiction, and hence $(S)$ must be true.

Choose $J, G_{0}$ and $G_{1}$ according to $(S)$. Considering the decomposition

$$
A=\prod_{i \in J} D_{i} \times \prod_{i \in I-J} D_{i},
$$

let $f$ be the projection of $A$ onto the factor $\prod_{i \in J} D_{i}$, and let $G^{*}$ be the image of $G_{1}$ under $f$. It follows from (2) that $f$ maps $G_{1}$ isomorphically onto $G^{*}$, and that

$$
G^{*}[p]=G_{1}[p]
$$

In particular, $G^{*}$ is torsion-complete. Furthermore, if $x \in G^{*}[p]$, then $x=f(x)$, and the height of $x$ in $G^{*}$ is at least as large as the height of $x$ in $G_{1}$. Since $G_{1}$ is a pure subgroup of $A$, it follows by 10.3 that $G^{*}$ is a pure subgroup of $A$. Thus, by $10.10, G^{*}$ is a factor of $A$, and consequently

$$
\prod_{i \in J} D_{i}=G^{*} \times H
$$

for some subgroup $H$. By the first part of the proof, $G^{*}$ has the finite exchange property, and thus there exist subgroups $E_{i} \subseteq D_{i}(i \in J)$ such that 


$$
\prod_{i \in J} D_{i}=G^{*} \times \prod_{i \in J} E_{i}
$$

Therefore

$$
A=G_{0} \times G_{1} \times C=G^{*} \times \prod_{i \in I} E_{i}
$$

where $E_{i}=D_{i}$ if $i \in I-J$. From (6) and this last decomposition we see that the direct product

$$
A^{\prime}=G_{1} \times \prod_{i \in I} E_{i},
$$

exists. Moreover, if $y \in G^{*}$ then there is an element $x \in G_{1}$ such that $y=f(x)$. Hence $x=y+z$ for some element $z \in \prod_{i \in I-J} D_{i}=\prod_{i \in I-J} E_{i}$, and we conclude that $y=x-z \in A^{\prime}$. This shows that $G^{*} \subseteq A^{\prime}$, and therefore $A^{\prime}=A$. Finally, $G_{0}$ is of bounded order and thus has the exchange property by 11.2. According to 3.9 we can therefore find subalgebras $F_{i} \subseteq E_{i}(i \in I)$ such that

$$
A=G_{0} \times G_{1} \times \prod_{i \in I} F_{i}=G \times \prod_{i \in I} F_{i} .
$$

Hence $G$ has the exchange property, and the proof of 11.4 is complete.

THEOREM 11.5. If $B$ is a binary algebra such that the reduced part of $B^{c}$ is a torsion group each primary component of which is torsion-complete, then $B$ has the exchange property.

Proof. This is an immediate consequence of 8.1, 11.1, 11.3 and 11.4, together with the introductory remarks of this section.

Combining 11.5 with $4.2,5.3$ and 7.1 , respectively, we obtain the following principal uniqueness and isomorphic refinement theorems for binary algebras.

THEOREM 11.6. If a binary algebra $A$ has two direct decompositions with countably many factors,

$$
A=B_{0} \times B_{1} \times B_{2} \times \cdots=C_{0} \times C_{1} \times C_{2} \times \cdots,
$$

where the reduced parts of all the groups $B_{i}^{c}$ and $C_{j}^{c}$ are torsion groups with torsion-complete primary components, then these two direct decompositions of $A$ have centrally isomorphic refinements.

CoROLlary 11.7. If $A$ is a binary algebra such that the reduced part of $A^{c}$ is a torsion group with torsion-complete primary components, then any two countable direct decompositions of $A$ have centrally isomorphic refinements. 
THEOREM 11.8. If a binary algebra $A$ has a direct decomposition

$$
A=\prod_{i \in I} B_{i}
$$

where, for each $i \in I$, the reduced part of $B_{i}^{c}$ is a torsion group with torsion-complete primary components, then any two direct decompositions of $A$ into indecomposable factors are centrally isomorphic.

THEOREM 11.9. If a binary algebra $A$ has a direct decomposition

$$
A=\prod_{i \in I} B_{i}
$$

where, for each $i \in I, B_{i}^{c}$ is countable and the reduced part of $B_{i}^{c}$ is a torsion group each primary component of which is of bounded order, then any two direct decompositions of $A$ have centrally isomorphic refinements.

A final theorem describes a class of binary algebras with uncountable centers having the isomorphic refinement property.

THEOREM 11.10. If $A$ is a binary algebra such that the maximal divisible subgroup of $A^{c}$ is countable and the reduced part of $A^{c}$ is a torsion group each primary component of which is a torsion-complete group with countable basic subgroups, then any two direct decompositions of $A$ have centrally isomorphic refinements.

\section{Proof. Suppose}

$$
A=\prod_{i \in I} B_{i}=\prod_{\jmath \in J} C_{j}
$$

Since the maximal divisible subgroup of $A^{c}$ is countable and the basic subgroups of each primary component of the reduced part of $A^{c}$ are countable, it follows that there exists a countable subset $I^{\prime}$ of $I$ such that $B_{i}^{c}=\{0\}$ for each $i \in I-I^{\prime}$. The factor $\prod_{i \in I-I^{\prime}} B_{i}$ has the exchange property, and hence there are subalgebras $D_{j}, D_{j}^{\prime}(j \in J)$ such that $C_{j}=D_{j} \times D_{J}^{\prime}$ and

$$
A=\prod_{i \in I-I^{\prime}} B_{i} \times \prod_{j \in J} D_{j}
$$

Consequently

$$
\prod_{i \in I-I^{\prime}} B_{i} \cong \prod_{j \in J}^{c} D_{j}^{\prime},
$$

and, as $\prod_{i \in I-I^{\prime}} B_{i}^{c}=\{0\}$, we infer by 2.19 that

$$
\prod_{i \in I^{\prime}} B_{i}=\prod_{j \in J} D_{j}
$$


Repeating the argument above for the factor $\Pi_{j \in J} D_{j}$, there is a countable subset $J^{\prime}$ of $J$ such that $D_{j}^{c}=\{0\}$ for each $j \in J-J^{\prime}$, and there are subalgebras $E_{i}, E_{i}^{\prime}\left(i \in I^{\prime}\right)$ such that $B_{i}=E_{i} \times E_{i}^{\prime}$ and

$$
\begin{aligned}
\prod_{j \in J-J^{\prime}} D_{j} & \cong \prod_{i \in I^{\prime}} E_{i}^{\prime}, \\
\prod_{j \in J^{\prime}} D_{j} & =\prod_{i \in I^{\prime}} E_{i} .
\end{aligned}
$$

The pairs of decompositions (2) and (4) each have centrally isomorphic refinements by 11.9 , and the decompositions (5) have centrally isomorphic refinements by 11.7. Therefore the original decompositions (1) have centrally isomorphic refinements, and the proof is complete.

12. Counterexamples and open problems. This final section contains two examples that yield negative answers to some questions related to the results in this paper. A number of unsolved problems suggested by our investigations are also mentioned.

In 3.10 it was shown that if an algebra $B$ is a direct product of finitely many subalgebras each of which has the exchange property, then $B$ has the exchange property. The first example shows that this result cannot be extended to products of infinitely many subalgebras. In fact, the example shows that if $B$ is an abelian $p$-group such that

$$
B=B_{1} \times B_{2} \times B_{3} \times \cdots
$$

where, for $k=1,2,3 \cdots, B_{k}$ is a cyclic group of order $p^{k}$, then $B$ does not have the 2-exchange property. Thus the simplest unbounded abelian $p$-group fails to have the exchange property.

Let

$$
A=\prod_{k=1}^{\infty}\left[u_{k}\right] \times \prod_{k=1}^{\infty}\left[v_{k}\right]
$$

where, for $k=1,2,3, \cdots,\left[u_{k}\right]$ and $\left[v_{k}\right]$ are cyclic groups of order $p^{k}$. Also, let

$$
\begin{aligned}
B & =\prod_{k=1}^{\infty}\left[u_{k}+p v_{k+1}\right], & C & =\prod_{k=1}^{\infty}\left[v_{k}\right], \\
D_{1} & =\prod_{k=1}^{\infty}\left[v_{k}+p u_{k+1}\right], & D_{2} & =\prod_{k=1}^{\infty}\left[u_{k}\right] .
\end{aligned}
$$

It is easy to check that

$$
A=B \times C=D_{1} \times D_{2},
$$

and in order to prove that $B$ does not have the 2-exchange property it is sufficient to show that the assumption that 


$$
A=B \times E_{1} \times E_{2}, \quad E_{1} \subseteq D_{1}, \quad E_{2} \subseteq D_{2}
$$

leads to a contradiction.

Assume that (1) holds. Since $A$ is a direct product of finite groups, it and all its direct factors have the unique factorization property. Inasmuch as $C \cong E_{1} \times E_{2}$, this implies that for each positive integer $k$ only one of the groups $E_{1}$ and $E_{2}$ has a cyclic factor of order $p^{k}$. Observing that

$$
B \times E_{2} \subseteq B \times D_{2}=B \times p C,
$$

we have $v_{k}=b+p c+e$ where $b \in B, c \in C, b+p c \in B \times E_{2}$, and $e \in E_{1}$. Using the fact that $B \times C$ exists we see that, for $r=1,2,3, \cdots, k-1$, the element $p^{r}\left(v_{k}-b\right)=p^{r+1} c+p^{r} e$ has height $r$, and hence the height of $p^{r} e$ is also $r$. Since $p^{k} e=0$, this shows that $[e]$ is a pure subgroup of $E_{1}$, and hence a factor of $E_{1}$, of order $p^{k}$. Consequently $E_{2}$ cannot have a direct factor of order $p^{k}$, and since this is true for every positive integer $k$, we infer that $E_{2}=\{0\}$, and hence $A=B \times E_{1} \subseteq B \times D_{1}$. But it is easy to see that neither $u_{1}$ nor $v_{1}$ belongs to $B \times D_{1}$, and we have thus arrived at a contradiction.

In 8.1 it was shown that if the center of an algebra $B$ has the exchange property, then $B$ has the exchange property. Our second example shows that the converse of this result is false. For this purpose we construct a group $B$ such that

(i) $B^{c}$ is an infinite cyclic group.

(ii) The commutator subgroup of $B$ equals $B,[B, B]=B$.

First observe that this does in fact imply that $B$ has the required properties. In fact, suppose $B^{c}=[u]$ and let $A=B^{c} \times C$ where $C=[v]$ is also an infinite cyclic group. Also let $D_{1}=[2 u+3 v]$ and $D_{2}=[3 u+5 v]$. Then $A=D_{1} \times D_{2}$. Since $B^{c} \times D_{1}=B^{c} \times[3 v] \neq A$ and $B^{c} \times D_{2}=B^{c} \times[5 v] \neq A$, we see that $B^{c}$ does not have the 2-exchange property. On the other hand, suppose $A$ is any algebra containing $B$ as a subalgebra, and suppose $C$ and $D_{i}(i \in I)$ are subalgebras of $A$ such that

$$
A=B \times C=\prod_{i \in I} D_{i} .
$$

Let $g$ and $h_{i}$ be the projections of $A$ onto $C$ and $D_{i}$ induced by these two direct decompositions of $A$. Then $g h_{i}$ maps $B$ homomorphically into the center of $C$, whence it follows by (ii) that, for each $b \in B$, $g h_{i}(b)=0$ or, equivalently, $h_{i}(b) \in B$. Thus, for each $i \in I, h_{i}$ maps $B$ into $B \cap D_{i}$, and we infer that

$$
B=\prod_{i \in I}\left(B \cap D_{i}\right) .
$$


It follows by the modular law that for each $i \in I$ there exists a subalgebra $E_{i} \subseteq D_{i}$ such that $D_{i}=\left(B \cap D_{i}\right) \times E_{i}$, and we conclude that

$$
A=B \times \prod_{i \in I} E_{i} \text {. }
$$

Hence $B$ has the exchange property.

In order to construct a group having the properties (i) and (ii) we proceed as follows. For $n=2,3,4, \cdots$ let $H_{n}$ be the group of all $n$ by $n$ matrices of determinant 1 over a field of characteristic 0 that contains a primitive $n$th root of unity. Then the center of $H_{n}$ contains a cyclic group of order $n$, and the commutator subgroup of $H_{n}$ equals $H_{n}$. The Cartesian product $H$ of $H_{2}, H_{3}, H_{4}, \cdots$ therefore has the properties that its center contains an infinite cyclic group and that the commutator subgroup of $H$ is equal to $H$. We now take for $B$ a free amalgamated product of two isomorphic copies $B_{1}$ and $B_{2}$ of $H$, with amalgamated subgroup $Z=B_{1} \cap B_{2}$ an infinite cyclic group contained in the centers of both $B_{1}$ and $B_{2}$. It is known that $B^{c}=B_{i}^{c} \cap B_{2}^{c}$, so that $B^{c}$ is in this case the infinite cyclic group $Z$. Thus (i) holds, and it is obvious that (ii) is also satisfied.

The most interesting unsolved problem suggested by the results in this paper is whether in Theorem 7.1 the assumption of countably generated centers is needed. Specifically, is it true that if an algebra $A$ is a direct product of subalgebras each of which has the exchange property, then any two direct decompositions of $A$ have isomorphic refinements? Even if the answer is negative, one might hope for an affirmative answer in special cases, such as for groups whose centers are of bounded order. Of course, if the answer should turn out to be affirmative, then this would include Theorems 4.2, 5.3 and 7.1 as special cases.

Another problem concerns the relation of the finite exchange property and the exchange property: Is the exchange property always implied by the finite exchange property? In connection with Theorem 7.1 it would be particularly interesting to know whether for an algebra $B$ with a countable generated center the finite exchange property implies the $\boldsymbol{S}_{0}$-exchange property (and therefore the exchange property). It is not hard to show that for such an algebra $B$ the condition

$$
A=B \times C=D_{0} \times D_{1} \times D_{2} \times \cdots
$$

implies that

$$
A=B \times E_{0} \times E_{1} \times E_{2} \times \cdots
$$

where each of the factors $E_{k}$ is a subalgebra of the finite product $D_{0} \times D_{1} \times \cdots \times D_{k}$, but we do not know whether the factors $E_{k}$ can be replaced by subalgebras of the factors $D_{k}$. 
Theorem 8.3 raises the problem of determining those abelian operator groups that have the exchange property. In this regard the following question seems particularly relevant: Is it true that if an abelian operator group satisfies the minimal condition, then it has the exchange property? For ordinary reduced abelian groups the results in $\S 11$ apply only to groups with no elements of infinite height. It would be of interest to know whether the class of all reduced primary abelian groups having the exchange property contains any groups with (nonzero) elements of infinite height.

\section{BIBLIOGRAPHY}

1. R. Baer, Direct decompositions, Trans. Amer. Math. Soc., 62 (1947), 62-98.

2. — Direct decompositions into in finitely many summands, Trans. Amer. Math. Soc., 64 (1948), 519-551.

3. G. Birkhoff, Lattice theory, Amer. Math., Soc. Colloquium Publications, vol. 25, rev. ed., New York, 1948.

4. P. Crawley, Direct decompositions with finite dimensional factors, Pacific J. Math., 12 (1962), 457-468.

5. L. Fuchs, Abelian groups, Budapest, 1958.

6. B. Jónsson and A. Tarski, Direct decompositions of finite algebraic systems, Notre Dame Mathematical Lectures, no. 5, 1947.

7. I. Kaplansky, In finite abelian groups, Ann Arbor, 1956.

8. W. Specht, Gruppentheorie, Berlin, Gottingen and Heidelberg, 1956. 



\section{PACIFIC JOURNAL OF MATHEMATICS}

\section{EDITORS}

\author{
Robert Osserman \\ Stanford University \\ Stanford, California
M. G. Arsove
University of Washington
Seattle 5 , Washington

\author{
J. DugundJI \\ University of Southern California \\ Los Angeles 7, California
}

Lowell J. Paige

University of California

Los Angeles 24, California

\section{ASSOCIATE EDITORS}
E. F. BECKENBACH
B. H. NeumanN
F. WOLF
K. YOSIDA

\section{SUPPORTING INSTITUTIONS}

\author{
UNIVERSITY OF BRITISH COLUMBIA \\ CALIFORNIA INSTITUTE OF TECHNOLOGY \\ UNIVERSITY OF CALIFORNIA \\ MONTANA STATE UNIVERSITY \\ UNIVERSITY OF NEVADA \\ NEW MEXICO STATE UNIVERSITY \\ OREGON STATE UNIVERSITY \\ UNIVERSITY OF OREGON \\ OSAKA UNIVERSITY \\ UNIVERSITY OF SOUTHERN CALIFORNIA
}

\author{
STANFORD UNIVERSITY \\ UNIVERSITY OF TOKYO \\ UNIVERSITY OF UTAH \\ WASHINGTON STATE UNIVERSITY \\ UNIVERSITY OF WASHINGTON \\ * * * * \\ AMERICAN MATHEMATICAL SOCIETY \\ CALIFORNIA RESEARCH CORPORATION \\ SPACE TECHNOLOGY LABORATORIES \\ NAVAL ORDNANCE TEST STATION
}

Mathematical papers intended for publication in the Pacific Journal of Mathematics should by typewritten (double spaced), and on submission, must be accompanied by a separate author's résumé. Manuscripts may be sent to any one of the four editors. All other communications to the editors should be addressed to the managing editor, L. J. Paige at the University of California, Los Angeles 24, California.

50 reprints per author of each article are furnished free of charge; additional copies may be obtained at cost in multiples of 50 .

The Pacific Journal of Mathematics is published quarterly, in March, June, September, and December. Effective with Volume 13 the price per volume (4 numbers) is $\$ 18.00$; single issues, $\$ 5.00$. Special price for current issues to individual faculty members of supporting institutions and to individual members of the American Mathematical Society: $\$ 8.00$ per volume; single issues $\$ 2.50$. Back numbers are available.

Subscriptions, orders for back numbers, and changes of address should be sent to Pacific Journal of Mathematics, 103 Highland Boulevard, Berkeley 8, California.

Printed at Kokusai Bunken Insatsusha (International Academic Printing Co., Ltd.), No. 6, 2-chome, Fujimi-cho, Chiyoda-ku, Tokyo, Japan.

PUBLISHED BY PACIFIC JOURNAL OF MATHEMATICS, A NON-PROFIT CORPORATION

The Supporting Institutions listed above contribute to the cost of publication of this Journal, but they are not owners or publishers and have no responsibility for its content or policies. 


\section{Pacific Journal of Mathematics}

\section{Vol. 14, No. $3 \quad$ July, 1964}

Erik Balslev and Theodore William Gamelin, The essential spectrum of a class of ordinary differential operators . . . . . . . . . . . . . . . . . . . .

James Henry Bramble and Lawrence Edward Payne, Bounds for derivatives in

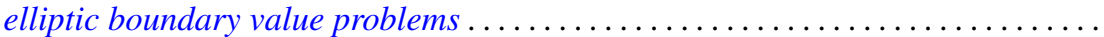

Hugh D. Brunk, Integral inequalities for functions with nondecreasing

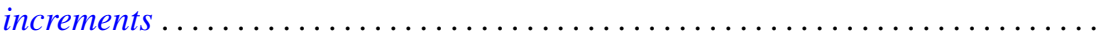

William Edward Christilles, A result concerning integral binary quadratic

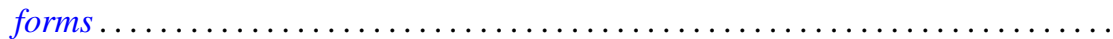

Peter Crawley and Bjarni Jónsson, Refinements for infinite direct decompositions of

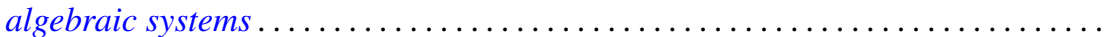

Don Deckard and Carl Mark Pearcy, On continuous matrix-valued functions on a Stonian space.

Raymond Frank Dickman, Leonard Rubin and P. M. Swingle, Another

characterization of the $n$-sphere and related results $\ldots \ldots \ldots \ldots \ldots \ldots$

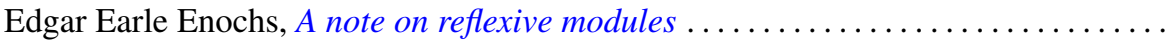

Vladimir Filippenko, On the reflection of harmonic functions and of solutions of the

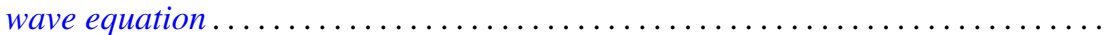

Derek Joseph Haggard Fuller, Mappings of bounded characteristic into arbitrary

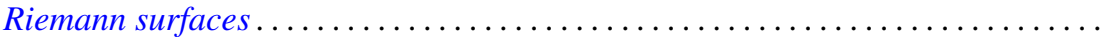
895

Curtis M. Fulton, Clifford vectors . . . . . . . . . . . . . . . . . . . . . . . . . . . . . . 917

Irving Leonard Glicksberg, Maximal algebras and a theorem of Radó . .

919

Kyong Taik Hahn, Minimum problems of Plateau type in the Bergman metric

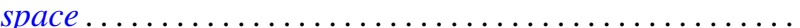

A. Hayes, A representation theory for a class of partially ordered rings...

J. M. C. Joshi, On a generalized Stieltjes trasform

J. M. C. Joshi, Inversion and representation theorems for a generalized Laplace transform ...

Eugene Kay McLachlan, Extremal elements of the convex cone $B_{n}$ of functions ...

Robert Alan Melter, Contributions to Boolean geometry of p-rings ...

James Ronald Retherford, Basic sequences and the Paley-Wiener criterion . . . . . . . 1019

Dallas W. Sasser, Quasi-positive operators. .

Oved Shisha, On the structure of infrapolynomials with prescribed coefficients ..

Oved Shisha and Gerald Thomas Cargo, On comparable means

Maurice Sion, A characterization of weak ${ }^{*}$ convergence ........

Morton Lincoln Slater and Robert James Thompson, A permanent inequality for

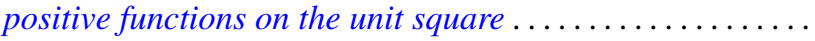

David A. Smith, On fixed points of automorphisms of classical Lie algebras ...

Sherman K. Stein, Homogeneous quasigroups ................

J. L. Walsh and Oved Shisha, On the location of the zeros of some infrapolynomials with prescribed coefficients .

Ronson Joseph Warne, Homomorphisms of $d$-simple inverse semigroups with identity . 